\title{
Adverse Cardiovascular Complications Following Prescription of Programmed Cell Death 1 (PD-1) and Programmed Cell Death Ligand 1 (PD-L1) Inhibitors: A Propensity-Score Matched Cohort Study With Competing Risk Analysis
}

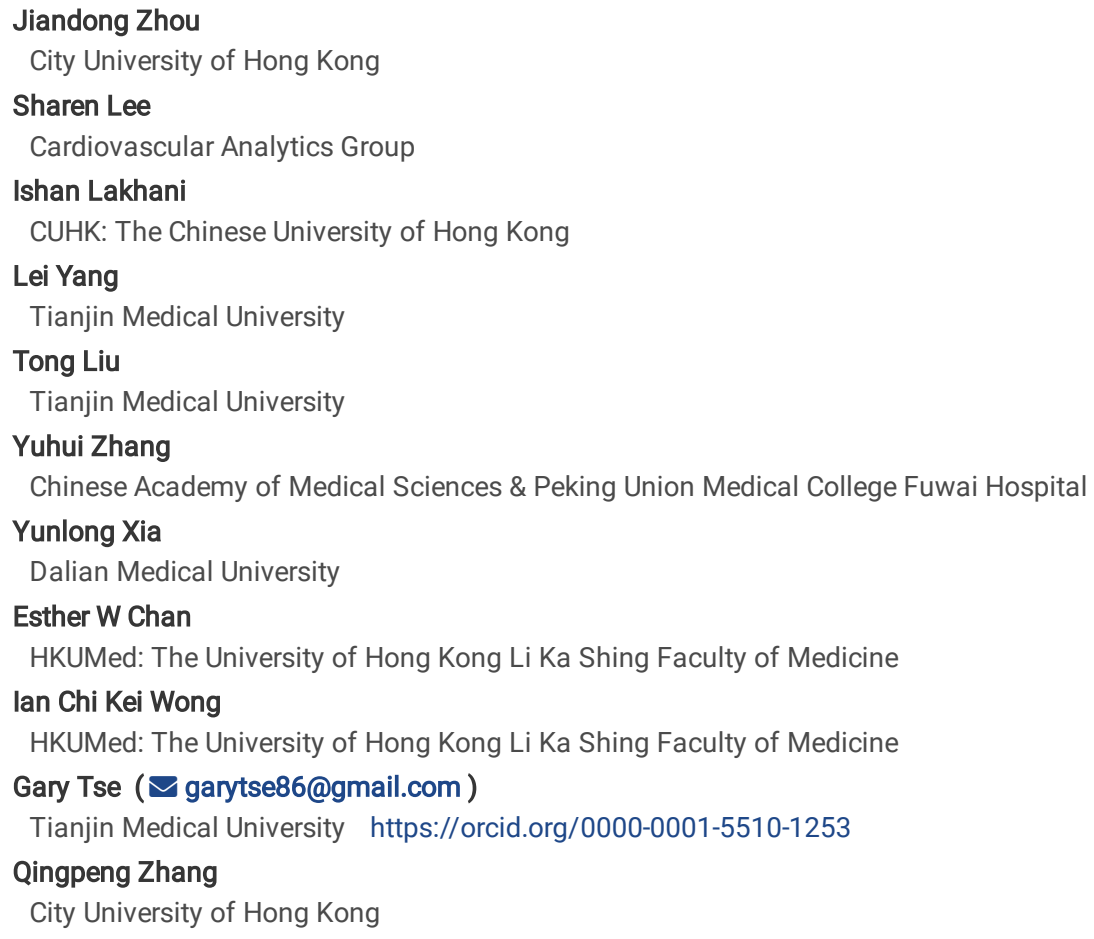

Research

Keywords: Programmed death, PD-1 treatment, hospitalizations, atrial fibrillation, cardiac complications

Posted Date: August 26th, 2021

DOI: https://doi.org/10.21203/rs.3.rs-829375/v1

License: @ (1) This work is licensed under a Creative Commons Attribution 4.0 International License. Read Full License

Version of Record: A version of this preprint was published at Cardio-Oncology on March 17th, 2022. See the published version at https://doi.org/10.1186/s40959-021-00128-5. 


\section{Abstract}

Background: Programmed death-1 (PD-1) and programmed death- ligand 1 (PD-L1) inhibitors, such as pembrolizumab, nivolumab and atezolizumab, are major classes of immune checkpoint inhibitors that are increasingly used for cancer treatment. However, their use is associated with adverse cardiovascular events. We examined the incidence of new-onset cardiac complications in patients receiving PD-1 or PD-L1 inhibitors.

Methods: Patients receiving PD-1 or PD-L1 inhibitors since their launch up to $31^{\text {st }}$ December 2019 at the Hospital Authority of Hong Kong, without pre-existing cardiac complications were included. The primary outcome was a composite of incident heart failure, acute myocardial infarction, atrial fibrillation or atrial flutter with the last follow-up date of $31^{\text {st }}$ December 2020. Propensity score matching between PD-L1 inhibitor use and PD-1 inhibitor use with a 1:2 ratio for patient demographics, past comorbidities and non-PD-1/PD-L1 medications was performed.

Results: A total of 1959 patients were included. Over a median follow-up of 247 days (interquartile range [IQR]: $72-506), 320$ (incidence rate [IR]: 16.31\%) patients met the primary outcome after PD-1/PD-L1 treatment: 244 (IR: 12.57\%) with heart failure, 38 (IR: 1.93\%) with acute myocardial infarction, 54 (IR: 2.75\%) with atrial fibrillation, 6 (IR: $0.31 \%$ ) with atrial flutter. Compared with PD-1 inhibitor treatment, PD-L1 inhibitor treatment was significantly associated with a lower risk of composite outcome both before (hazard ratio [HR]: 0.32, 95\% Cl: [0.18-0.59], P value=0.0002) and after matching (HR: 0.34, 95\% Cl: [0.180.65], P value=0.001), and lower all-cause mortality risk before matching (HR: 0.77, 95\% Cl: [0.64-0.93], P value=0.0078) and after matching (HR: 0.80, 95\% Cl: [0.65-1.00], $P$ value=0.0463). Patients who developed cardiac complications had shorter average readmission intervals and a higher number of hospitalizations after treatment with PD-1/PD-L1 inhibitors both before and after matching ( $\mathrm{P}$ value $<0.0001)$. Competing risk analysis with cause-specific hazard and subdistribution hazard models, and multiple approaches based on the propensity score all confirmed these observations.

Conclusions: Compared with PD-1 treatment, PD-L1 treatment was significantly associated with lower risk of new onset cardiac composite outcome and allcause mortality both before and after propensity score matching.

\section{Introduction}

The programmed death-1 (PD-1)/programmed death-ligand 1 (PD-L1) pathway is one of the major immune checkpoints for mitigating the immune response to prevent autoimmunity. However, cancer cells often devise strategies to hijack these mechanisms to evade anti-tumor immunity. In this regard, inhibitors of PD-1 (e.g., pembrolizumab, nivolumab, cemiplimab) and PD-L1 (e.g. atezolizumab, avelumab, durvalumab) have shown clinical efficacies against different types of solid tumors, including melanoma, non-small cell lung cancer (NSCLC), urothelial carcinoma and bladder cancer. Pembrolizumab is also the first agent to receive a "pan-cancer" approval by the United States Food and Drug Administration (FDA) for the treatment of unresectable or metastatic solid tumors that have high microsatellite instability or mismatch repair deficiency.

Despite their treatment efficacy in clinical oncology, immune-related adverse events associated with the use of immune checkpoint inhibitors (ICls) are now increasingly recognized (1-4). Adverse events include atherosclerosis, colitis, hepatitis, adrenocorticotropic hormone insufficiency, hypothyroidism, type 1 diabetes mellitus, and acute kidney injury (5-7). To this end, cardiovascular complications are estimated to constitute approximately $2 \%$ of ICl-related adverse drug reactions (8). The commonest is myocarditis, but other cardiovascular abnormalities reported are left ventricular dysfunction, acute myocardial infarction (AMI), cardiac arrhythmias and heart failure (9). These cardiovascular complications typically present with clinical heterogeneity, and in turn account for the high morbidity and mortality rates observed in such patient cohorts. Whilst cardiotoxicity is being documented with an increasing frequency, their cumulative incidence rates remain largely unexplored. In this territory-wide study, we examined the incidence of cardiovascular events of incident heart failure, acute myocardial infarction, atrial fibrillation or atrial flutter in cancer patients receiving PD-1 or PD-L1 inhibitors.

\section{Methods Study Population}

This study was approved by The Joint Chinese University of Hong Kong - New Territories East Cluster Clinical Research Ethics Committee. Patients receiving PD-1 or PD-L1 inhibitors since their launch up to 31 st December 2020 at the Hospital Authority of Hong Kong, without pre-existing cardiac complications were included. Patient data were obtained using the electronic health record database, which is connected to the territory-wide Clinical Data Analysis and Reporting System (CDARS). The system is an integrative centralized platform that permits the extraction of clinical data for analysis and reporting. The system attributes each patient a unique reference identification number, allowing for the retrieval of comprehensive medical records, including disease diagnoses, clinical comorbidities, laboratory parameters and operative procedures. Patients or the public were not involved in any aspect of this study. The system has been previously used by both our team and other teams in Hong Kong (10-12).

\section{Patient Data}

The following clinical data were extracted: patient characteristics, including demographic details (baseline age and gender), specific pre-existing comorbidities before drug prescriptions, laboratory examinations (including complete blood counts, biochemical tests, lipid and glycemic profiles) were extracted. Past comorbidities from January 1st, 2013 to December 31st, 2020 were extracted, and categorized into hypertension, liver diseases, hip fractures/accident falls, renal diseases, diabetes mellitus, maligt dysrhythmia, chronic obstructive pulmonary disease, ischemic heart disease, peripheral vascular disease, endocrine diseases, gastrointestinal diseases, and stroke/transient ischemic attack. The International Classification of Disease, Ninth Edition (ICD-9) codes that were used to extract the specific comorbidities and outcomes are included in Supplementary Table 2 . The overall dosage and accumulative duration for patients with new-onset cardiac complications are reported

\section{Primary outcomes on follow-up}


The primary outcome was a composite of incident heart failure, acute myocardial infarction, atrial fibrillation, and atrial flutter. The follow-up period was defined as the first PD-1/PD-L1 prescription date until the primary endpoint or death occurred, or until the end date of August 31st, 2020, whichever was earlier.

\section{Statistical analysis}

Continuous variables were presented as median (95\% confidence interval [CI] or interquartile range [IQR]) and categorical variables were presented as count

(\%). The Mann- Whitney U test was used to compare continuous variables. The $\chi^{2}$ test with Yates' correction was used for $2 \times 2$ contingency data, and Pearson's $\chi^{2}$ test was used for contingency data for variables with more than two categories. The patients with PD-L1 were matched with PD1 controls through propensity score matching of 1:2 ratio, based on patient demographics, Charlson standard comorbidity index, past comorbidities, and non-PD-1/PD-L1 medications. Negligible post-weighting intergroup standardized mean difference (SMD) was defined as SMD $<0.2$. To identify the important predictors associated with new-onset cardiac complications of patients after PD-1/PD-L1 treatment, univariate Cox regression was used to calculate hazard ratios (HRs) and $95 \%$ Cls. In addition to propensity score matching, the following approaches based on the propensity scores were employed: propensity score stratification (13), inverse probability weighting (14), and high-dimensional propensity score adjustment (15). Paired hospitalization characteristics of patients before and after treatment were compared both in the unmatched and matched cohorts. A two-sided a of $<0.05$ was considered statistically significant. Statistical analyses were performed using RStudio software (Version: 1.1.456) and Python (Version: 3.6).

\section{Results}

\section{Baseline characteristics}

Initially, 2426 cancer patients receiving PD-1/PD-L1 inhibitors were identified (Fig. 1). In total 1959 patients remained in the study cohort after excluding 433 patients with prior cardiac complications and 34 patients who received both PD1 and PDL1 treatments. Propensity score matching with 1:2 ratio between PD1 and PD-L1 inhibitor use based on demographics, Charlson standard comorbidity index, prior comorbidities, and non-PD-1/PD-L1 medications was performed. This yielded a matched cohort of 663 patients (Table 1).

Table 1. Clinical characteristics of patients with PD-1 use and PD-L1 use before and after 1:2 propensity score matching.

* for SMD 0.2

APTT: applied partial thromboplastin test; PD-1: Programmed death 1 inhibitors; PD-L1: programmed death 1 ligand inhibitors 
Before matching

All $(\mathrm{N}=1959)$

Mean(SD);N or Count(\%)

PD-L1 users ( $\mathrm{N}=221)$

Mean(SD);N or Count(\%)

PD-1 users $(\mathrm{N}=1738)$

Mean(SD);N or Count(\%)

SMD

After 1:2 matching

\section{Characteristics}

Adverse events

All-caus

mortality

Composite

outcome

Heart failure

$244(12.45 \%)$

Acute myocardial $\quad 38(1.93 \%)$

infarction

Atrial fibrillation $\quad 54(2.75 \%)$

Atrial flutter $\quad 6(0.30 \%)$

$115(52.03 \%)$

Demographics

Male gender

Female gender

Baseline age,

years

$<40$

$[40,50)$

$[50-60)$

[60-70)

$[70-80)$

$>=80$

Past

comorbidities

Charlson standard

comorbidity index

Hypertension

Liver diseases

1341(68.45\%)

618(31.54\%)

61.0(13.7);n=1959

151(7.70\%)

194(9.90\%)

$481(24.55 \%)$

631(32.21\%)

391(19.95\%)

$111(5.66 \%)$

6.1(3.3); $n=1959$

256(13.06\%)

193(9.85\%)

77(3.93\%)

fractures/accident

falls

Renal diseases

Diabetes mellitus

Maligt

dysrhythmia

Chronic

obstructive

pulmonary

disease

Ischemic hear

disease

Peripheral

vascular disease

Endocrine

diseases

Gastrointestinal

diseases

Stroke/transient ischemic attack

\section{Hospitalization}

Average 68.5(194.8);n=1876

$61(3.11 \%)$

$11(0.56 \%)$

$548(27.97 \%)$

$1412(72.07 \%)$

$80(4.08 \%)$
$11(4.97 \%)$

$10(4.52 \%)$

$0(0.00 \%)$

$1(0.45 \%)$

$0(0.00 \%)$

\begin{tabular}{lll}
\hline $1204(69.27 \%)$ & $0.36 *$ & $425(64.10 \%)$ \\
\hline $309(17.77 \%)$ & $0.41^{*}$ & $91(13.72 \%)$ \\
\hline $234(13.46 \%)$ & $0.32 *$ & $75(11.31 \%)$ \\
\hline $38(2.18 \%)$ & $0.21 *$ & $8(1.20 \%)$ \\
\hline $53(3.04 \%)$ & 0.2 & $8(1.20 \%)$ \\
\hline $6(0.34 \%)$ & 0.08 & $2(0.30 \%)$ \\
\hline
\end{tabular}

$0.15 \quad 498(75.11 \%)$

$0.15165(24.88 \%)$

$56(25.33 \%)$

$1176(67.66 \%)$

$562(32.33 \%)$

$0.19 \quad 63.0(10.2) ; n=663$

60.7(14.1);n=1738

$0.22^{*} \quad 20(3.01 \%)$

$0.1743(6.48 \%)$

$0.04 \quad 155(23.37 \%)$

0.26 * 288(43.43\%)

$0.05133(20.06 \%)$

$0.16 \quad 24(3.61 \%)$

105(6.04\%)

6.0(3.3); $n=1738$

$0.16 \quad 6.5(3.1) ; n=663$

6

29(13.12\%)

227(13.06\%)

185(10.64\%)

$0 \quad 88(13.27 \%)$

$0.28^{*} \quad 24(3.61 \%)$

$13(5.88 \%)$

$64(3.68 \%)$

$0.1 \quad 37(5.58 \%)$

$29(13.12 \%)$

263(15.13\%)

$0.06 \quad 85(12.82 \%)$

$0.04 \quad 60(9.04 \%)$

$0.12 \quad 2(0.30 \%)$

$0(0.00 \%)$

136(7.82\%)

$12(0.69 \%)$

$0.02 \quad 6(0.90 \%)$

$13(0.74 \%)$

$56(3.22 \%)$

$0.06 \quad 15(2.26 \%)$

5

$1(0.45 \%)$

$10(0.57 \%)$

$0.023(0.45 \%)$

492(28.30\%)

$0.07 \quad 166(25.03 \%)$

0.30 * $551(83.10 \%)$

184(83.25\%)

$1228(70.65 \%)$

$0.03 \quad 24(3.61 \%)$

$72(4.14 \%)$

$8(3.61 \%)$

53.3(112.0);n=215

70.5(203.0);n=1661

$0.1 \quad 65.3(202.3) ; n=647$

5 
readmission

\begin{tabular}{|c|c|c|c|c|c|c|}
\hline $\begin{array}{l}\text { Total episode } \\
\text { number }\end{array}$ & $14.2(13.9) ; n=1876$ & $12.2(8.9) ; n=215$ & $14.5(14.4) ; n=1661$ & 0.19 & $13.8(10.9) ; n=647$ & 1 \\
\hline $\begin{array}{l}\text { Overall hospital } \\
\text { stay, days }\end{array}$ & $35.2(38.2) ; n=1876$ & $25.5(22.4) ; n=215$ & $36.5(39.6) ; n=1661$ & $0.34^{\star}$ & $32.9(31.3) ; n=647$ & 2 \\
\hline \multicolumn{7}{|l|}{ Medications } \\
\hline PD-L1 v.s. PD-1 & $221(11.28 \%)$ & $221(100.00 \%)$ & $0(0.00 \%)$ & inf* & $221(33.33 \%)$ & 2 \\
\hline $\begin{array}{l}\text { PD-L1 } \\
\text { expenditure, HKD }\end{array}$ & 98251.0(98697.8);n=221 & 98251.0(98697.8);n=221 & - & - & 98251.0(98697.8);n=221 & 9 \\
\hline $\begin{array}{l}\text { Total PD-L1 dose } \\
\text { amount, mg }\end{array}$ & $12644.1(27488.6) ; n=221$ & $12644.1(27488.6) ; n=221$ & - & - & 12644.1(27488.6);n=221 & 1 \\
\hline $\begin{array}{l}\text { PD-L1 inhibitors } \\
\text { duration, days }\end{array}$ & $176.0(200.9) ; n=221$ & $176.0(200.9) ; n=221$ & - & - & $176.0(200.9) ; n=221$ & 1 \\
\hline PD-1 expenditure & $193878.8(291968.7) ; n=1750$ & $333403.4(284147.8) ; n=12$ & $192915.5(291869.7) ; n=1738$ & $0.49 *$ & $203782.5(259059.2) ; n=454$ & 3 \\
\hline $\begin{array}{l}\text { Total PD-1 dose } \\
\text { amount (MG) }\end{array}$ & 2817.9(10492.2);n=1750 & $1450.0(1049.5) ; n=12$ & 2827.3(10527.4);n=1738 & 0.18 & $2454.7(5152.0) ; n=454$ & 1 \\
\hline $\begin{array}{l}\text { PD-1 inhibitors } \\
\text { duration, days }\end{array}$ & 202.7(237.7);n=1750 & $383.3(270.7) ; n=12$ & 201.5(237.0);n=1738 & $0.71^{\star}$ & 207.7(234.7);n=454 & 3 \\
\hline Anticoagulants & $1108(56.55 \%)$ & $120(54.29 \%)$ & $988(56.84 \%)$ & 0.05 & $362(54.60 \%)$ & 1 \\
\hline Steroids & $1108(56.55 \%)$ & $120(54.29 \%)$ & $988(56.84 \%)$ & 0.05 & $362(54.60 \%)$ & 1 \\
\hline
\end{tabular}

\section{Inflammatory \\ subclinical \\ biomarkers}

\begin{tabular}{|c|c|c|c|c|c|c|}
\hline $\begin{array}{l}\text { Neutrophil-to- } \\
\text { lymphocyte ratio }\end{array}$ & $4.6(6.4) ; n=1952$ & $4.5(9.5) ; n=221$ & $4.6(5.9) ; n=1731$ & 0.01 & $4.3(6.5) ; n=663$ & 4 \\
\hline $\begin{array}{l}\text { Platelet-to- } \\
\text { lymphocyte ratio }\end{array}$ & $212.3(243.9) ; n=1953$ & 203.9(188.4);n=221 & $213.4(250.1) ; n=1732$ & 0.04 & $210.2(304.3) ; n=663$ & 2 \\
\hline $\begin{array}{l}\text { Aspartate } \\
\text { transaminase-to- } \\
\text { alanine } \\
\text { transaminase } \\
\text { ratio }\end{array}$ & $1.8(3.6) ; n=1308$ & $1.1(0.5) ; n=128$ & $1.9(3.8) ; n=1180$ & $0.29 *$ & $1.5(2.1) ; n=405$ & 1 \\
\hline $\begin{array}{l}\text { Triglyceride } \\
\text { glucose index }\end{array}$ & $7.1(0.6) ; n=580$ & $7.07(0.65) ; n=67$ & $7.06(0.62) ; n=513$ & 0.02 & $7.0(0.6) ; n=207$ & 7 \\
\hline $\begin{array}{l}\text { Urea-to-creatinine } \\
\text { ratio }\end{array}$ & $73.1(40.8) ; n=1937$ & $68.3(23.3) ; n=221$ & $73.8(42.4) ; n=1716$ & 0.16 & $69.5(29.2) ; n=659$ & 6 \\
\hline $\begin{array}{l}\text { Monocyte-to- } \\
\text { lymphocyte ratio }\end{array}$ & $0.4(0.5) ; n=1950$ & $0.4(0.5) ; n=221$ & $0.5(0.5) ; n=1729$ & 0.03 & $0.4(0.4) ; n=662$ & 0 \\
\hline
\end{tabular}

Complete blood

\section{counts}

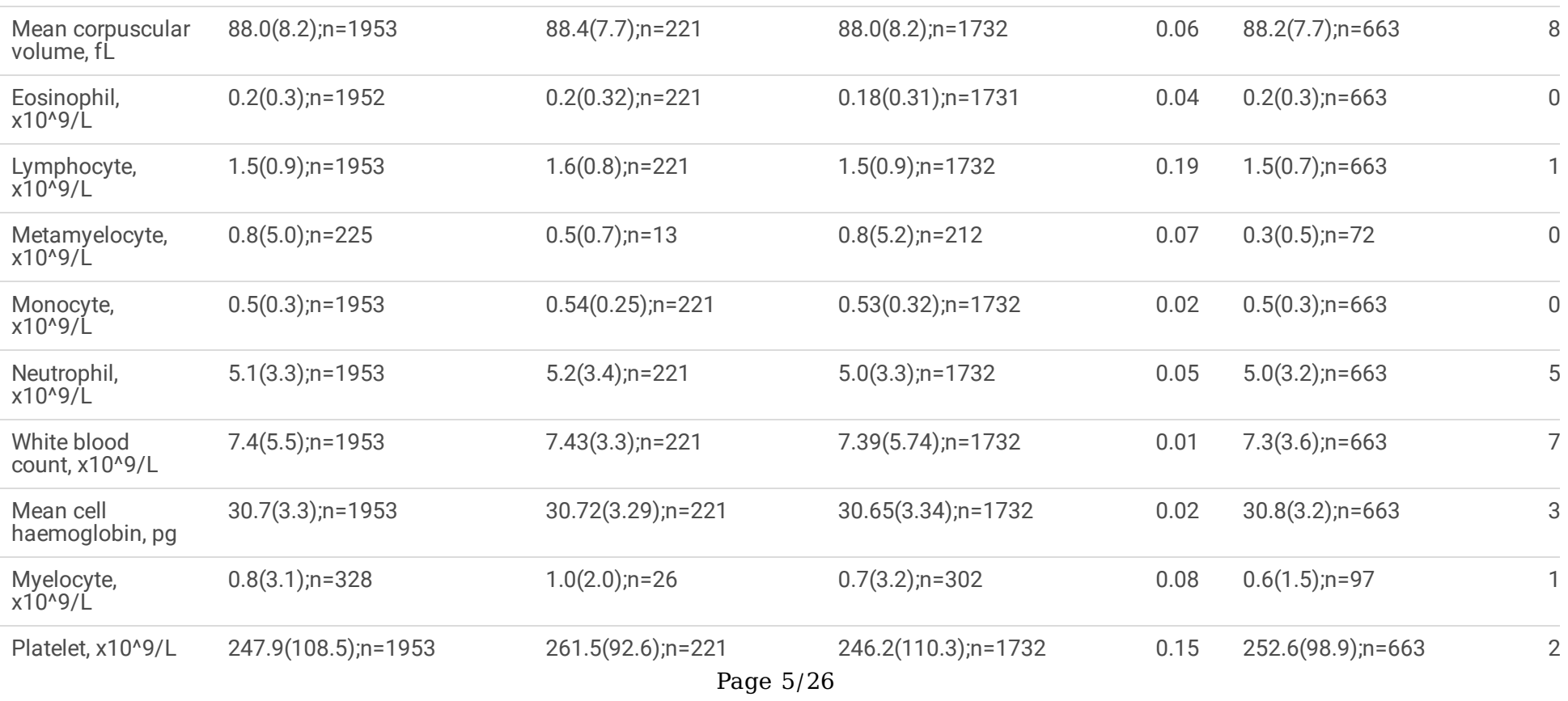




\begin{tabular}{|c|c|c|c|c|c|c|}
\hline $\begin{array}{l}\text { Red blood count, } \\
\times 10^{\wedge} 12 / \mathrm{L}\end{array}$ & $4.4(0.7) ; n=1953$ & $4.6(0.6) ; n=221$ & $4.4(0.7) ; n=1732$ & $0.27^{*}$ & $4.5(0.7) ; n=663$ & 4 \\
\hline Hematocrit, L/L & $0.4(0.1) ; n=1895$ & $0.4(0.05) ; n=208$ & $0.38(0.06) ; n=1687$ & $0.37 *$ & $0.4(0.1) ; n=626$ & 0 \\
\hline \multicolumn{7}{|l|}{$\begin{array}{l}\text { Renal and liver } \\
\text { functions }\end{array}$} \\
\hline $\begin{array}{l}\text { K/Potassium, } \\
\mathrm{mmol} / \mathrm{L}\end{array}$ & $4.1(0.4) ; n=1941$ & $4.14(0.43) ; n=221$ & $4.12(0.44) ; n=1720$ & 0.04 & $4.1(0.4) ; n=661$ & 4 \\
\hline Urate, $\mathrm{mmol} / \mathrm{L}$ & $0.3(0.1) ; n=627$ & $0.33(0.1) ; n=43$ & $0.33(0.14) ; n=584$ & 0.03 & $0.3(0.1) ; n=186$ & 0 \\
\hline Albumin, g/L & $39.2(5.9) ; n=1939$ & $39.8(5.4) ; n=220$ & $39.1(6.0) ; n=1719$ & 0.11 & $39.5(5.4) ; n=660$ & 3 \\
\hline $\begin{array}{l}\mathrm{Na} / \text { Sodium, } \\
\mathrm{mmol} / \mathrm{L}\end{array}$ & 139.1(3.8);n=1941 & $139.4(3.1) ; n=221$ & 139.1(3.9);n=1720 & 0.09 & 139.2(3.4);n=661 & 1 \\
\hline Urea, mmol/L & $5.5(2.4) ; n=1937$ & $5.3(1.8) ; n=221$ & $5.6(2.5) ; n=1716$ & 0.11 & $5.3(1.8) ; n=659$ & 5 \\
\hline Protein, $\mathrm{g} / \mathrm{L}$ & $72.1(12.8) ; n=1853$ & 71.3(16.3);n=207 & $72.2(12.3) ; n=1646$ & 0.06 & $71.7(14.4) ; n=624$ & 7 \\
\hline Bilirubin, umol/L & 13.0(25.3);n=1941 & $9.5(6.1) ; n=221$ & 13.4(26.7);n=1720 & 0.2 & 11.3(19.1);n=661 & 9 \\
\hline Creatinine, umol/L & $84.9(62.4) ; n=1952$ & $83.0(37.0) ; n=221$ & $85.1(64.9) ; n=1731$ & 0.04 & $84.8(64.0) ; n=662$ & 8 \\
\hline SD of creatinine & $35.5(103.7) ; n=1942$ & 29.8(93.9);n=220 & $36.2(104.9) ; n=1722$ & 0.06 & 29.7(82.3);n=658 & 2 \\
\hline $\begin{array}{l}\text { Aspartate } \\
\text { transaminase, U/L }\end{array}$ & $54.4(104.9) ; n=1371$ & $28.3(21.8) ; n=142$ & $57.5(110.1) ; n=1229$ & $0.37 *$ & $41.2(67.2) ; n=443$ & 2 \\
\hline $\begin{array}{l}\text { SD of aspartate } \\
\text { transaminase }\end{array}$ & $59.6(189.6) ; n=1250$ & $28.6(101.7) ; n=122$ & $62.9(196.5) ; n=1128$ & $0.22^{*}$ & $36.5(84.3) ; n=409$ & 2 \\
\hline $\begin{array}{l}\text { Alkaline } \\
\text { phosphatase, U/L }\end{array}$ & 108.6(111.6);n=1941 & $85.2(36.4) ; n=221$ & $111.6(117.5) ; \mathrm{n}=1720$ & $0.3^{*}$ & $102.1(99.9) ; n=661$ & 8 \\
\hline $\begin{array}{l}\text { SD of alkaline } \\
\text { phosphatase }\end{array}$ & 64.8(95.1);n=1928 & $39.6(75.3) ; n=220$ & $68.0(96.9) ; n=1708$ & $0.33^{*}$ & $58.3(89.9) ; n=655$ & 3 \\
\hline $\begin{array}{l}\text { Alanine } \\
\text { transaminase, } \mathrm{U} / \mathrm{L}\end{array}$ & $36.6(55.7) ; n=1876$ & $28.2(25.1) ; n=207$ & $37.6(58.3) ; n=1669$ & $0.21^{*}$ & $31.4(29.5) ; n=623$ & 2 \\
\hline $\begin{array}{l}\text { SD of alanine } \\
\text { transaminase }\end{array}$ & $36.4(84.7) ; n=1855$ & $22.9(50.7) ; n=201$ & $38.0(87.8) ; n=1654$ & $0.21^{*}$ & $28.2(51.6) ; n=612$ & 2 \\
\hline \multicolumn{7}{|l|}{$\begin{array}{l}\text { Lipid, iron and } \\
\text { calcium profile }\end{array}$} \\
\hline $\begin{array}{l}\text { Total iron-binding } \\
\text { capacity, L }\end{array}$ & 41.0(12.3);n=103 & $39.1(11.1) ; n=5$ & $41.1(12.4) ; n=98$ & 0.16 & $42.1(12.7) ; n=22$ & 3 \\
\hline $\begin{array}{l}\text { VitaminB12, } \\
\mathrm{pmol} / \mathrm{L}\end{array}$ & $453.2(327.2) ; n=49$ & $447.7(265.6) ; n=3$ & $453.6(333.2) ; n=46$ & 0.02 & 401.2(305.7);n=17 & 4 \\
\hline Folate, $\mathrm{ng} / \mathrm{mL}$ & $21.7(10.2) ; n=69$ & $16.0(3.7) ; n=3$ & $22.0(10.3) ; n=66$ & $0.78^{*}$ & $20.4(10.4) ; n=19$ & 1 \\
\hline Ferritin, pmol/L & $2546.7(4740.6) ; n=86$ & $728.2(816.5) ; \mathrm{n}=8$ & $2733.2(4936.4) ; n=78$ & $0.57 *$ & $1941.1(2987.1) ; n=22$ & 7 \\
\hline Calcium, mmol/L & $2.3(0.2) ; n=1081$ & $2.32(0.13) ; n=82$ & 2.32(0.16);n=999 & 0.04 & $2.3(0.1) ; n=340$ & 2 \\
\hline SD of calcium & $0.1(0.1) ; n=987$ & $0.07(0.04) ; n=73$ & $0.1(0.06) ; n=914$ & $0.49 *$ & $0.1(0.1) ; n=314$ & 0 \\
\hline $\begin{array}{l}\text { Phosphate, } \\
\mathrm{mmol} / \mathrm{L}\end{array}$ & $1.1(0.2) ; n=948$ & $1.08(0.18) ; n=61$ & $1.06(0.22) ; n=887$ & 0.11 & $1.1(0.2) ; n=286$ & 1 \\
\hline SD of phosphate & $0.2(0.1) ; n=785$ & $0.1(0.1) ; n=44$ & $0.2(0.1) ; n=741$ & $0.41^{*}$ & $0.2(0.1) ; n=241$ & 0 \\
\hline Inorganic, mmol/L & $6.4(8.7) ; n=259$ & $4.1(5.4) ; n=24$ & $6.6(8.9) ; n=235$ & $0.34^{*}$ & $6.5(10.2) ; n=79$ & 4 \\
\hline SD of inorganic & $2.1(3.5) ; n=113$ & $0.5(0.7) ; n=13$ & $2.3(3.7) ; n=100$ & $0.7^{*}$ & $0.8(1.4) ; n=41$ & 0 \\
\hline \multicolumn{7}{|l|}{$\begin{array}{l}\text { Glycemic and } \\
\text { clotting profile }\end{array}$} \\
\hline $\begin{array}{l}\text { Triglyceride, } \\
\mathrm{mmol} / \mathrm{L}\end{array}$ & $1.4(1.1) ; n=598$ & $1.5(1.0) ; n=68$ & $1.4(1.1) ; n=530$ & 0.08 & $1.5(1.2) ; n=214$ & 1 \\
\hline SD of triglyceride & $0.4(0.5) ; n=294$ & $0.4(0.43) ; n=30$ & $0.35(0.54) ; n=264$ & 0.1 & $0.4(0.6) ; n=90$ & 0 \\
\hline Glucose, mmol/L & $6.3(2.3) ; n=1773$ & $6.4(2.7) ; n=206$ & $6.2(2.2) ; n=1567$ & 0.09 & $6.3(2.2) ; n=608$ & 6 \\
\hline SD of glucose & $1.5(1.3) ; n=1638$ & 1.4(1.3);n=194 & $1.5(1.3) ; n=1444$ & 0.09 & $1.5(1.4) ; n=564$ & 1 \\
\hline $\mathrm{HbA} 1 \mathrm{c}, \mathrm{g} / \mathrm{dL}$ & $12.8(2.0) ; n=1953$ & $13.3(1.7) ; n=221$ & $12.8(2.0) ; n=1732$ & $0.31 *$ & $13.1(1.9) ; n=663$ & 1 \\
\hline SD of $\mathrm{HbA} 1 \mathrm{c}$ & $1.3(0.5) ; n=1947$ & $1.31(0.54) ; n=220$ & $1.29(0.54) ; n=1727$ & 0.05 & $1.3(0.5) ; n=660$ & 1 \\
\hline
\end{tabular}




\begin{tabular}{|c|c|c|c|c|c|c|}
\hline $\begin{array}{l}\text { High sensitive } \\
\text { troponin-I, ng/L }\end{array}$ & $363.3(10967.7) ; \mathrm{n}=1141$ & $12.7(31.8) ; n=122$ & $405.3(11605.6) ; n=1019$ & 0.05 & $35.5(314.8) ; n=372$ & 1 \\
\hline $\begin{array}{l}\text { SD of high } \\
\text { sensitive troponin- } \\
\text { I }\end{array}$ & $124.0(1328.2) ; n=755$ & $18.2(42.4) ; n=82$ & $136.9(1406.3) ; n=673$ & 0.12 & $34.7(207.0) ; n=245$ & 1 \\
\hline APTT, second & $30.8(5.5) ; n=694$ & $29.1(3.5) ; n=46$ & $30.9(5.6) ; n=648$ & $0.39 *$ & $30.4(3.9) ; n=217$ & 2 \\
\hline SD of APTT & $2.6(3.1) ; n=510$ & $1.9(2.3) ; n=26$ & $2.6(3.2) ; n=484$ & $0.28 *$ & $2.1(1.8) ; n=152$ & 1 \\
\hline $\begin{array}{l}\text { Lactate } \\
\text { dehydrogenase, } \\
\text { U/L }\end{array}$ & $324.1(422.0) ; n=1431$ & $315.6(534.6) ; n=145$ & $325.1(407.6) ; n=1286$ & 0.02 & $302.1(354.0) ; n=466$ & 3 \\
\hline $\begin{array}{l}\text { SD of lactate } \\
\text { dehydrogenase }\end{array}$ & $155.2(397.1) ; \mathrm{n}=1140$ & 115.6(203.9);n=102 & 159.1(411.1);n=1038 & 0.13 & 121.3(206.3);n=365 & 1 \\
\hline $\begin{array}{l}\text { Total cholesterol, } \\
\mathrm{mmol} / \mathrm{L}\end{array}$ & $4.6(1.1) ; n=599$ & $4.9(1.0) ; n=68$ & $4.5(1.1) ; n=531$ & $0.29 *$ & $4.6(1.1) ; n=214$ & 4 \\
\hline $\begin{array}{l}\text { SD of total } \\
\text { cholesterol }\end{array}$ & $0.5(0.4) ; n=295$ & $0.54(0.43) ; n=31$ & $0.48(0.44) ; n=264$ & 0.14 & $0.5(0.5) ; n=93$ & 0 \\
\hline $\begin{array}{l}\text { Low-density } \\
\text { lipoprotein, } \\
\text { mmol/L }\end{array}$ & $2.6(0.9) ; n=583$ & $2.9(0.9) ; n=65$ & $2.6(0.9) ; n=518$ & $0.34^{*}$ & $2.7(1.0) ; \mathrm{n}=208$ & 2 \\
\hline $\begin{array}{l}\text { SD of low-density } \\
\text { lipoprotein }\end{array}$ & $0.4(0.4) ; n=283$ & $0.5(0.4) ; n=29$ & $0.4(0.4) ; n=254$ & 0.18 & $0.4(0.4) ; n=88$ & 0 \\
\hline $\begin{array}{l}\text { High-density } \\
\text { lipoprotein, } \\
\text { mmol/L }\end{array}$ & $1.3(0.4) ; n=593$ & $1.32(0.4) ; n=67$ & $1.34(0.4) ; n=526$ & 0.04 & $1.3(0.4) ; n=212$ & 1 \\
\hline $\begin{array}{l}\text { SD of high-density } \\
\text { lipoprotein }\end{array}$ & $0.1(0.1) ; n=280$ & $0.12(0.09) ; n=30$ & $0.14(0.14) ; n=250$ & 0.17 & $0.2(0.1) ; n=87$ & 0 \\
\hline
\end{tabular}

Propensity score matching in 1:2 ratio between PD-1 users and PD-L1 users using the nearest neighbor search strategy was used. The results of logistics regression for potential confounders used in propensity score calculations, balance between groups, and estimations of bootstrapped standard error are shown in Supplementary Tables 3, 4 and 5, respectively. Distributions of propensity scores before and after matching are shown in Supplementary Fig. 1. These results indicate that the covariates between the groups are balanced after matching.

\section{Adverse cardiovascular outcomes on follow-up and their significant predictors}

In the matched cohort, 425 (IR: 64.10\%) patients died and 91 (IR: 13.72\%) developed new onset cardiac composite outcome. Amongst the latter, 75 (IR: 11.31\%) developed heart failure, 8 (IR: 1.20\%) developed acute myocardial infarction, 8 (IR: 1.20\%) developed atrial fibrillation and 2 (IR: $0.30 \%$ ) developed atrial flutter. The incidence rate of the composite outcome was lower in the PD-L1 cohort than in the PD-1 cohort (7.0\% vs. 20.7\%; P<0.001).

In the 221 PD-L1 users, there were 11 patients (IR: 4.97\%) who developed the composite outcome, in which 10 (IR: $4.52 \%)$ with heart failure, 1 (0.45\%) with atrial fibrillation, but none with acute myocardial infarction or atrial flutter. In the 442 patients PD-1 users, 80 patients (IR: $18.09 \%$ ) developed the composite outcome. Of the latter group, 65 (IR: 14.70\%) developed heart failure, 8 (IR: 1.80\%) developed acute myocardial infarction, 7 (IR: $1.58 \%$ ) developed atrial fibrillation, and 2 (IR: $0.45 \%$ ) developed atrial flutter.

The breakdown on the individual adverse events is shown in Fig. 1 and the patient characteristics stratified by adverse cardiovascular outcomes are shown in Table 2 and Table 3. The Kaplan-Meier survival curves and the cumulative incidence curves of new onset cardiac complications and all-cause mortality in cancer patients stratified by PD-1 or PD-L1 inhibitor use before and after 1:2 propensity score matching were presented in Fig. 2 and Fig. 3, respectively. The baseline characteristics of the cohort stratified by mortality status are shown in Supplementary Table 6. 
Table 2

Clinical characteristics of patients who developed the composite outcome before and after 1:2 propensity score matchinc Before matching

After 1:2 matching

\begin{tabular}{|c|c|c|c|c|c|c|}
\hline \multirow[t]{2}{*}{ Characteristics } & All $(\mathrm{N}=1959)$ & PD-L1 users $(\mathrm{N}=221)$ & PD-1 users $(N=1738)$ & SMD & All $(N=663)$ & PD-L1 users $(\mathrm{N}=\mathbf{2 2 1}$ \\
\hline & $\begin{array}{l}\text { Mean(SD);N or } \\
\text { Count(\%) }\end{array}$ & $\begin{array}{l}\text { Mean(SD);N or } \\
\text { Count(\%) }\end{array}$ & $\begin{array}{l}\text { Mean(SD);N or } \\
\text { Count(\%) }\end{array}$ & & $\begin{array}{l}\text { Mean(SD);N or } \\
\text { Count(\%) }\end{array}$ & $\begin{array}{l}\text { Mean(SD);N or } \\
\text { Count(\%) }\end{array}$ \\
\hline \multicolumn{7}{|l|}{ Adverse events } \\
\hline $\begin{array}{l}\text { All-cause } \\
\text { mortality }\end{array}$ & $1319(67.33 \%)$ & $115(52.03 \%)$ & $1204(69.27 \%)$ & $0.36^{*}$ & $425(64.10 \%)$ & $115(52.03 \%)$ \\
\hline $\begin{array}{l}\text { Composite } \\
\text { outcome }\end{array}$ & $320(16.33 \%)$ & $11(4.97 \%)$ & $309(17.77 \%)$ & $0.41^{*}$ & $91(13.72 \%)$ & $11(4.97 \%)$ \\
\hline Heart failure & $244(12.45 \%)$ & $10(4.52 \%)$ & $234(13.46 \%)$ & $0.32^{\star}$ & $75(11.31 \%)$ & $10(4.52 \%)$ \\
\hline $\begin{array}{l}\text { Acute myocardial } \\
\text { infarction }\end{array}$ & $38(1.93 \%)$ & $0(0.00 \%)$ & $38(2.18 \%)$ & $0.21^{*}$ & $8(1.20 \%)$ & $0(0.00 \%)$ \\
\hline Atrial fibrillation & $54(2.75 \%)$ & $1(0.45 \%)$ & $53(3.04 \%)$ & 0.2 & $8(1.20 \%)$ & $1(0.45 \%)$ \\
\hline Atrial flutter & $6(0.30 \%)$ & $0(0.00 \%)$ & $6(0.34 \%)$ & 0.08 & $2(0.30 \%)$ & $0(0.00 \%)$ \\
\hline \multicolumn{7}{|l|}{ Demographics } \\
\hline Male gender & $1341(68.45 \%)$ & $165(74.66 \%)$ & $1176(67.66 \%)$ & 0.15 & $498(75.11 \%)$ & $165(74.66 \%)$ \\
\hline Female gender & 618(31.54\%) & $56(25.33 \%)$ & $562(32.33 \%)$ & 0.15 & $165(24.88 \%)$ & $56(25.33 \%)$ \\
\hline $\begin{array}{l}\text { Baseline age, } \\
\text { years }\end{array}$ & $61.0(13.7) ; n=1959$ & $63.1(10.2) ; n=221$ & $60.7(14.1) ; n=1738$ & 0.19 & $63.0(10.2) ; \mathrm{n}=663$ & $63.1(10.2) ; n=221$ \\
\hline$<40$ & 151(7.70\%) & $7(3.16 \%)$ & $144(8.28 \%)$ & $0.22^{\star}$ & $20(3.01 \%)$ & $7(3.16 \%)$ \\
\hline$[40,50)$ & 194(9.90\%) & $13(5.88 \%)$ & $181(10.41 \%)$ & 0.17 & $43(6.48 \%)$ & $13(5.88 \%)$ \\
\hline$[50-60)$ & $481(24.55 \%)$ & $51(23.07 \%)$ & $430(24.74 \%)$ & 0.04 & $155(23.37 \%)$ & $51(23.07 \%)$ \\
\hline$[60-70)$ & $631(32.21 \%)$ & $96(43.43 \%)$ & $535(30.78 \%)$ & $0.26 *$ & $288(43.43 \%)$ & $96(43.43 \%)$ \\
\hline$[70-80)$ & 391(19.95\%) & $48(21.71 \%)$ & $343(19.73 \%)$ & 0.05 & $133(20.06 \%)$ & $48(21.71 \%)$ \\
\hline$>=80$ & $111(5.66 \%)$ & $6(2.71 \%)$ & $105(6.04 \%)$ & 0.16 & $24(3.61 \%)$ & $6(2.71 \%)$ \\
\hline \multicolumn{7}{|l|}{$\begin{array}{l}\text { Past } \\
\text { comorbidities }\end{array}$} \\
\hline $\begin{array}{l}\text { Charlson standard } \\
\text { comorbidity index }\end{array}$ & 6.1(3.3);n = 1959 & $6.5(3.1) ; n=221$ & $6.0(3.3) ; n=1738$ & 0.16 & $6.5(3.1) ; n=663$ & $6.53(3.1) ; n=221$ \\
\hline Hypertension & $256(13.06 \%)$ & $29(13.12 \%)$ & $227(13.06 \%)$ & 0 & $88(13.27 \%)$ & $29(13.12 \%)$ \\
\hline Liver diseases & 193(9.85\%) & $8(3.61 \%)$ & $185(10.64 \%)$ & $0.28^{\star}$ & $24(3.61 \%)$ & $8(3.61 \%)$ \\
\hline $\begin{array}{l}\text { Hip } \\
\text { fractures/accident } \\
\text { falls }\end{array}$ & 77(3.93\%) & $13(5.88 \%)$ & $64(3.68 \%)$ & 0.1 & $37(5.58 \%)$ & $13(5.88 \%)$ \\
\hline Renal diseases & $292(14.90 \%)$ & $29(13.12 \%)$ & $263(15.13 \%)$ & 0.06 & $85(12.82 \%)$ & $29(13.12 \%)$ \\
\hline Diabetes mellitus & $156(7.96 \%)$ & $20(9.04 \%)$ & $136(7.82 \%)$ & 0.04 & $60(9.04 \%)$ & $20(9.04 \%)$ \\
\hline $\begin{array}{l}\text { Maligt } \\
\text { dysrhythmia }\end{array}$ & $12(0.61 \%)$ & $0(0.00 \%)$ & $12(0.69 \%)$ & 0.12 & $2(0.30 \%)$ & $0(0.00 \%)$ \\
\hline $\begin{array}{l}\text { Chronic } \\
\text { obstructive } \\
\text { pulmonary } \\
\text { disease }\end{array}$ & $15(0.76 \%)$ & $2(0.90 \%)$ & $13(0.74 \%)$ & 0.02 & $6(0.90 \%)$ & $2(0.90 \%)$ \\
\hline $\begin{array}{l}\text { Ischemic heart } \\
\text { disease }\end{array}$ & $61(3.11 \%)$ & $5(2.26 \%)$ & $56(3.22 \%)$ & 0.06 & $15(2.26 \%)$ & $5(2.26 \%)$ \\
\hline $\begin{array}{l}\text { Peripheral } \\
\text { vascular disease }\end{array}$ & $11(0.56 \%)$ & $1(0.45 \%)$ & $10(0.57 \%)$ & 0.02 & $3(0.45 \%)$ & $1(0.45 \%)$ \\
\hline $\begin{array}{l}\text { Endocrine } \\
\text { diseases }\end{array}$ & $548(27.97 \%)$ & $56(25.33 \%)$ & $492(28.30 \%)$ & 0.07 & $166(25.03 \%)$ & $56(25.33 \%)$ \\
\hline
\end{tabular}

* for SMD 0.2; \# indicates the difference between patients with/without the composite outcome

APTT: applied partial thromboplastin test; PD-1: Programmed death 1 inhibitors; PD-L1: programmed death 1 ligand inhibitors 


\begin{tabular}{|c|c|c|c|c|c|c|}
\hline & Before matching & & & & After 1:2 matching & \\
\hline $\begin{array}{l}\text { Gastrointestinal } \\
\text { diseases }\end{array}$ & $1412(72.07 \%)$ & 184(83.25\%) & $1228(70.65 \%)$ & $0.30 *$ & $551(83.10 \%)$ & 184(83.25\%) \\
\hline $\begin{array}{l}\text { Stroke/transient } \\
\text { ischemic attack }\end{array}$ & $80(4.08 \%)$ & $8(3.61 \%)$ & $72(4.14 \%)$ & 0.03 & $24(3.61 \%)$ & $8(3.61 \%)$ \\
\hline \multicolumn{7}{|l|}{ Hospitalization } \\
\hline $\begin{array}{l}\text { Average } \\
\text { readmission }\end{array}$ & $68.5(194.8) ; n=1876$ & $53.3(112.0) ; n=215$ & 70.5(203.0); $\mathrm{n}=1661$ & 0.1 & $65.3(202.3) ; n=647$ & $53.3(112.0) ; n=215$ \\
\hline $\begin{array}{l}\text { Total episode } \\
\text { number }\end{array}$ & 14.2(13.9);n = 1876 & $12.2(8.9) ; n=215$ & $14.5(14.4) ; n=1661$ & 0.19 & $13.8(10.9) ; n=647$ & $12.2(8.9) ; n=215$ \\
\hline $\begin{array}{l}\text { Overall hospital } \\
\text { stay, days }\end{array}$ & $35.2(38.2) ; n=1876$ & $25.5(22.4) ; \mathrm{n}=215$ & $36.5(39.6) ; n=1661$ & $0.34^{*}$ & $32.9(31.3) ; \mathrm{n}=647$ & $25.5(22.4) ; n=215$ \\
\hline \multicolumn{7}{|l|}{ Medications } \\
\hline PD-L1 v.s. PD-1 & $221(11.28 \%)$ & $221(100.00 \%)$ & $0(0.00 \%)$ & inf* & 221(33.33\%) & $221(100.00 \%)$ \\
\hline $\begin{array}{l}\text { PD-L1 } \\
\text { expenditure, HKD }\end{array}$ & $\begin{array}{l}98251.0(98697.8) ; n= \\
221\end{array}$ & $\begin{array}{l}98251.0(98697.8) ; n= \\
221\end{array}$ & - & - & $\begin{array}{l}98251.0(98697.8) ; n= \\
221\end{array}$ & $\begin{array}{l}98251.0(98697.8) ; n= \\
221\end{array}$ \\
\hline $\begin{array}{l}\text { Total PD-L1 dose } \\
\text { amount, mg }\end{array}$ & $\begin{array}{l}12644.1(27488.6) ; n= \\
221\end{array}$ & $\begin{array}{l}12644.1(27488.6) ; n= \\
221\end{array}$ & - & - & $\begin{array}{l}12644.1(27488.6) ; n= \\
221\end{array}$ & $\begin{array}{l}12644.1(27488.6) ; n= \\
221\end{array}$ \\
\hline $\begin{array}{l}\text { PD-L1 inhibitors } \\
\text { duration, days }\end{array}$ & $176.0(200.9) ; n=221$ & $176.0(200.9) ; n=221$ & - & - & $176.0(200.9) ; n=221$ & $176.0(200.9) ; \mathrm{n}=221$ \\
\hline PD-1 expenditure & $\begin{array}{l}\text { 193878.8(291968.7);n } \\
=1750\end{array}$ & $\begin{array}{l}333403.4(284147.8) ; n \\
=12\end{array}$ & $\begin{array}{l}\text { 192915.5(291869.7);n } \\
=1738\end{array}$ & $0.49 *$ & $\begin{array}{l}203782.5(259059.2) ; n \\
=454\end{array}$ & $\begin{array}{l}333403.4(284147.8) ; \\
=12\end{array}$ \\
\hline $\begin{array}{l}\text { Total PD-1 dose } \\
\text { amount (MG) }\end{array}$ & $\begin{array}{l}2817.9(10492.2) ; n= \\
1750\end{array}$ & $1450.0(1049.5) ; n=12$ & $\begin{array}{l}2827.3(10527.4) ; n= \\
1738\end{array}$ & 0.18 & $\begin{array}{l}2454.7(5152.0) ; n= \\
454\end{array}$ & $1450.0(1049.5) ; \mathrm{n}=1 \%$ \\
\hline $\begin{array}{l}\text { PD-1 inhibitors } \\
\text { duration, days }\end{array}$ & 202.7(237.7);n= 1750 & $383.3(270.7) ; n=12$ & $201.5(237.0) ; n=1738$ & $0.71^{*}$ & $207.7(234.7) ; \mathrm{n}=454$ & $383.3(270.7) ; n=12$ \\
\hline Anticoagulants & $1108(56.55 \%)$ & $120(54.29 \%)$ & $988(56.84 \%)$ & 0.05 & $362(54.60 \%)$ & $120(54.29 \%)$ \\
\hline Steroids & $1108(56.55 \%)$ & $120(54.29 \%)$ & $988(56.84 \%)$ & 0.05 & $362(54.60 \%)$ & $120(54.29 \%)$ \\
\hline \multicolumn{7}{|l|}{$\begin{array}{l}\text { Inflammatory } \\
\text { subclinical } \\
\text { biomarkers }\end{array}$} \\
\hline $\begin{array}{l}\text { Neutrophil-to- } \\
\text { lymphocyte ratio }\end{array}$ & 4.6(6.4);n = 1952 & $4.5(9.5) ; n=221$ & $4.6(5.9) ; n=1731$ & 0.01 & $4.3(6.5) ; n=663$ & $4.5(9.5) ; n=221$ \\
\hline $\begin{array}{l}\text { Platelet-to- } \\
\text { lymphocyte ratio }\end{array}$ & 212.3(243.9);n = 1953 & $203.9(188.4) ; n=221$ & $213.4(250.1) ; n=1732$ & 0.04 & $210.2(304.3) ; n=663$ & $203.9(188.4) ; n=221$ \\
\hline $\begin{array}{l}\text { Aspartate } \\
\text { transaminase-to- } \\
\text { alanine } \\
\text { transaminase } \\
\text { ratio }\end{array}$ & $1.8(3.6) ; n=1308$ & $1.1(0.5) ; n=128$ & $1.9(3.8) ; n=1180$ & $0.29 *$ & $1.5(2.1) ; n=405$ & $1.1(0.5) ; n=128$ \\
\hline $\begin{array}{l}\text { Triglyceride } \\
\text { glucose index }\end{array}$ & $7.1(0.6) ; n=580$ & $7.07(0.65) ; \mathrm{n}=67$ & $7.06(0.62) ; \mathrm{n}=513$ & 0.02 & $7.0(0.6) ; n=207$ & $7.1(0.6) ; n=67$ \\
\hline $\begin{array}{l}\text { Urea-to-creatinine } \\
\text { ratio }\end{array}$ & $73.1(40.8) ; n=1937$ & $68.3(23.3) ; n=221$ & $73.8(42.4) ; n=1716$ & 0.16 & $69.5(29.2) ; n=659$ & $68.3(23.3) ; n=221$ \\
\hline $\begin{array}{l}\text { Monocyte-to- } \\
\text { lymphocyte ratio }\end{array}$ & $0.4(0.5) ; n=1950$ & $0.4(0.5) ; n=221$ & $0.5(0.5) ; n=1729$ & 0.03 & $0.4(0.4) ; n=662$ & $0.4(0.5) ; n=221$ \\
\hline \multicolumn{7}{|l|}{$\begin{array}{l}\text { Complete blood } \\
\text { counts }\end{array}$} \\
\hline $\begin{array}{l}\text { Mean corpuscular } \\
\text { volume, } \mathrm{fL}\end{array}$ & $88.0(8.2) ; n=1953$ & $88.4(7.7) ; n=221$ & $88.0(8.2) ; n=1732$ & 0.06 & $88.2(7.7) ; n=663$ & $88.4(7.7) ; n=221$ \\
\hline $\begin{array}{l}\text { Eosinophil, } \\
\text { x10^9/L }\end{array}$ & $0.2(0.3) ; \mathrm{n}=1952$ & $0.2(0.32) ; n=221$ & $0.18(0.31) ; n=1731$ & 0.04 & $0.2(0.3) ; n=663$ & $0.2(0.32) ; n=221$ \\
\hline $\begin{array}{l}\text { Lymphocyte, } \\
\times 10^{\wedge} 9 / \mathrm{L}\end{array}$ & $1.5(0.9) ; n=1953$ & $1.6(0.8) ; n=221$ & $1.5(0.9) ; \mathrm{n}=1732$ & 0.19 & $1.5(0.7) ; n=663$ & $1.6(0.8) ; n=221$ \\
\hline
\end{tabular}

* for SMD 0.2; \# indicates the difference between patients with/without the composite outcome

APTT: applied partial thromboplastin test; PD-1: Programmed death 1 inhibitors; PD-L1: programmed death 1 ligand inhibitors 


\begin{tabular}{|c|c|c|c|c|c|c|}
\hline & Before matching & & & & After 1:2 matching & \\
\hline 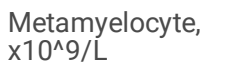 & $0.8(5.0) ; n=225$ & $0.5(0.7) ; n=13$ & $0.8(5.2) ; n=212$ & 0.07 & $0.3(0.5) ; n=72$ & $0.5(0.7) ; n=13$ \\
\hline $\begin{array}{l}\text { Monocyte, } \\
\times 10^{\wedge} 9 / L\end{array}$ & $0.5(0.3) ; n=1953$ & $0.54(0.25) ; n=221$ & $0.53(0.32) ; n=1732$ & 0.02 & $0.5(0.3) ; n=663$ & $0.54(0.25) ; n=221$ \\
\hline $\begin{array}{l}\text { Neutrophil, } \\
\times 10^{\wedge} 9 / \mathrm{L}\end{array}$ & $5.1(3.3) ; n=1953$ & $5.2(3.4) ; \mathrm{n}=221$ & $5.0(3.3) ; n=1732$ & 0.05 & $5.0(3.2) ; n=663$ & $5.2(3.4) ; n=221$ \\
\hline $\begin{array}{l}\text { White blood } \\
\text { count, } \times 10^{\wedge} 9 / \mathrm{L}\end{array}$ & $7.4(5.5) ; n=1953$ & $7.43(3.3) ; n=221$ & $7.39(5.74) ; n=1732$ & 0.01 & $7.3(3.6) ; n=663$ & $7.4(3.3) ; n=221$ \\
\hline $\begin{array}{l}\text { Mean cell } \\
\text { haemoglobin, pg }\end{array}$ & $30.7(3.3) ; n=1953$ & $30.72(3.29) ; n=221$ & $30.65(3.34) ; n=1732$ & 0.02 & $30.8(3.2) ; n=663$ & $30.7(3.3) ; n=221$ \\
\hline $\begin{array}{l}\text { Myelocyte, } \\
\times 10^{\wedge} 9 / \mathrm{L}\end{array}$ & $0.8(3.1) ; n=328$ & $1.0(2.0) ; n=26$ & $0.7(3.2) ; n=302$ & 0.08 & $0.6(1.5) ; n=97$ & $1.0(2.0) ; n=26$ \\
\hline Platelet, $\times 10^{\wedge} 9 / \mathrm{L}$ & $247.9(108.5) ; n=1953$ & $261.5(92.6) ; n=221$ & $246.2(110.3) ; n=1732$ & 0.15 & $252.6(98.9) ; n=663$ & $261.5(92.6) ; n=221$ \\
\hline $\begin{array}{l}\text { Red blood count, } \\
\text { x } 10^{\wedge} 12 / L\end{array}$ & $4.4(0.7) ; n=1953$ & $4.6(0.6) ; n=221$ & $4.4(0.7) ; n=1732$ & $0.27 *$ & $4.5(0.7) ; n=663$ & $4.6(0.6) ; n=221$ \\
\hline Hematocrit, L/L & $0.4(0.1) ; n=1895$ & $0.4(0.05) ; n=208$ & $0.38(0.06) ; n=1687$ & $0.37 *$ & $0.4(0.1) ; n=626$ & $0.4(0.05) ; n=208$ \\
\hline \multicolumn{7}{|l|}{$\begin{array}{l}\text { Renal and liver } \\
\text { functions }\end{array}$} \\
\hline $\begin{array}{l}\text { K/Potassium, } \\
\mathrm{mmol} / \mathrm{L}\end{array}$ & $4.1(0.4) ; n=1941$ & $4.14(0.43) ; n=221$ & $4.12(0.44) ; n=1720$ & 0.04 & $4.1(0.4) ; n=661$ & $4.14(0.43) ; n=221$ \\
\hline Urate, $\mathrm{mmol} / \mathrm{L}$ & $0.3(0.1) ; \mathrm{n}=627$ & $0.33(0.1) ; \mathrm{n}=43$ & $0.33(0.14) ; n=584$ & 0.03 & $0.3(0.1) ; \mathrm{n}=186$ & $0.33(0.1) ; \mathrm{n}=43$ \\
\hline Albumin, g/L & $39.2(5.9) ; n=1939$ & $39.8(5.4) ; n=220$ & 39.1(6.0);n = 1719 & 0.11 & $39.5(5.4) ; \mathrm{n}=660$ & $39.8(5.4) ; n=220$ \\
\hline $\begin{array}{l}\mathrm{Na} / \text { Sodium, } \\
\mathrm{mmol} / \mathrm{L}\end{array}$ & 139.1(3.8);n = 1941 & $139.4(3.1) ; n=221$ & $139.1(3.9) ; n=1720$ & 0.09 & $139.2(3.4) ; \mathrm{n}=661$ & $139.4(3.1) ; \mathrm{n}=221$ \\
\hline Urea, $\mathrm{mmol} / \mathrm{L}$ & $5.5(2.4) ; n=1937$ & $5.3(1.8) ; n=221$ & $5.6(2.5) ; n=1716$ & 0.11 & $5.3(1.8) ; n=659$ & $5.31(1.76) ; n=221$ \\
\hline Protein, $\mathrm{g} / \mathrm{L}$ & $72.1(12.8) ; n=1853$ & $71.3(16.3) ; n=207$ & $72.2(12.3) ; n=1646$ & 0.06 & $71.7(14.4) ; n=624$ & 71.3(16.3);n = 207 \\
\hline Bilirubin, umol/L & $13.0(25.3) ; n=1941$ & $9.5(6.1) ; n=221$ & $13.4(26.7) ; n=1720$ & 0.2 & $11.3(19.1) ; n=661$ & $9.5(6.1) ; n=221$ \\
\hline Creatinine, umol/L & $84.9(62.4) ; n=1952$ & $83.0(37.0) ; n=221$ & $85.1(64.9) ; n=1731$ & 0.04 & $84.8(64.0) ; \mathrm{n}=662$ & $83.0(37.0) ; \mathrm{n}=221$ \\
\hline SD of creatinine & $35.5(103.7) ; n=1942$ & 29.8(93.9);n = 220 & $36.2(104.9) ; n=1722$ & 0.06 & $29.7(82.3) ; n=658$ & 29.8(93.9);n = 220 \\
\hline $\begin{array}{l}\text { Aspartate } \\
\text { transaminase, U/L }\end{array}$ & $54.4(104.9) ; n=1371$ & $28.3(21.8) ; \mathrm{n}=142$ & $57.5(110.1) ; n=1229$ & $0.37 *$ & $41.2(67.2) ; \mathrm{n}=443$ & $28.3(21.8) ; n=142$ \\
\hline $\begin{array}{l}\text { SD of aspartate } \\
\text { transaminase }\end{array}$ & $59.6(189.6) ; n=1250$ & 28.6(101.7);n = 122 & $62.9(196.5) ; n=1128$ & $0.22^{*}$ & $36.5(84.3) ; n=409$ & $28.6(101.7) ; n=122$ \\
\hline $\begin{array}{l}\text { Alkaline } \\
\text { phosphatase, U/L }\end{array}$ & 108.6(111.6);n= 1941 & $85.2(36.4) ; n=221$ & $111.6(117.5) ; n=1720$ & $0.3^{*}$ & $102.1(99.9) ; n=661$ & $85.2(36.4) ; n=221$ \\
\hline $\begin{array}{l}\text { SD of alkaline } \\
\text { phosphatase }\end{array}$ & $64.8(95.1) ; n=1928$ & $39.6(75.3) ; n=220$ & $68.0(96.9) ; n=1708$ & $0.33^{*}$ & $58.3(89.9) ; n=655$ & $39.6(75.3) ; n=220$ \\
\hline $\begin{array}{l}\text { Alanine } \\
\text { transaminase, U/L }\end{array}$ & $36.6(55.7) ; n=1876$ & $28.2(25.1) ; n=207$ & $37.6(58.3) ; n=1669$ & $0.21^{\star}$ & $31.4(29.5) ; n=623$ & $28.2(25.1) ; \mathrm{n}=207$ \\
\hline $\begin{array}{l}\text { SD of alanine } \\
\text { transaminase }\end{array}$ & $36.4(84.7) ; n=1855$ & $22.9(50.7) ; n=201$ & $38.0(87.8) ; n=1654$ & $0.21^{\star}$ & $28.2(51.6) ; n=612$ & $22.9(50.7) ; n=201$ \\
\hline \multicolumn{7}{|l|}{$\begin{array}{l}\text { Lipid, iron and } \\
\text { calcium profile }\end{array}$} \\
\hline $\begin{array}{l}\text { Total iron-binding } \\
\text { capacity, L }\end{array}$ & $41.0(12.3) ; \mathrm{n}=103$ & $39.1(11.1) ; n=5$ & $41.1(12.4) ; n=98$ & 0.16 & $42.1(12.7) ; n=22$ & $39.1(11.1) ; n=5$ \\
\hline $\begin{array}{l}\text { VitaminB12, } \\
\mathrm{pmol} / \mathrm{L}\end{array}$ & $453.2(327.2) ; n=49$ & $447.7(265.6) ; n=3$ & $453.6(333.2) ; n=46$ & 0.02 & $401.2(305.7) ; n=17$ & $447.7(265.6) ; n=3$ \\
\hline Folate, ng/mL & $21.7(10.2) ; n=69$ & $16.0(3.7) ; n=3$ & $22.0(10.3) ; n=66$ & $0.78^{\star}$ & $20.4(10.4) ; n=19$ & $16.0(3.7) ; n=3$ \\
\hline Ferritin, pmol/L & $2546.7(4740.6) ; n=86$ & $728.2(816.5) ; n=8$ & $2733.2(4936.4) ; n=78$ & $0.57^{*}$ & $1941.1(2987.1) ; n=22$ & $728.2(816.5) ; n=8$ \\
\hline Calcium, mmol/L & $2.3(0.2) ; n=1081$ & $2.32(0.13) ; n=82$ & $2.32(0.16) ; n=999$ & 0.04 & $2.3(0.1) ; n=340$ & $2.32(0.13) ; n=82$ \\
\hline
\end{tabular}

* for SMD 0.2; \# indicates the difference between patients with/without the composite outcome

APTT: applied partial thromboplastin test; PD-1: Programmed death 1 inhibitors; PD-L1: programmed death 1 ligand inhibitors 


\begin{tabular}{|c|c|c|c|c|c|c|}
\hline & Before matching & & & & After $1: 2$ matching & \\
\hline SD of calcium & $0.1(0.1) ; n=987$ & $0.07(0.04) ; n=73$ & $0.1(0.06) ; n=914$ & $0.49 *$ & $0.1(0.1) ; n=314$ & $0.07(0.04) ; n=73$ \\
\hline $\begin{array}{l}\text { Phosphate, } \\
\mathrm{mmol} / \mathrm{L}\end{array}$ & $1.1(0.2) ; n=948$ & $1.08(0.18) ; n=61$ & $1.06(0.22) ; n=887$ & 0.11 & $1.1(0.2) ; n=286$ & $1.08(0.18) ; \mathrm{n}=61$ \\
\hline SD of phosphate & $0.2(0.1) ; n=785$ & $0.1(0.1) ; n=44$ & $0.2(0.1) ; \mathrm{n}=741$ & $0.41^{*}$ & $0.2(0.1) ; n=241$ & $0.1(0.1) ; n=44$ \\
\hline Inorganic, mmol/L & $6.4(8.7) ; n=259$ & $4.1(5.4) ; n=24$ & $6.6(8.9) ; n=235$ & $0.34^{\star}$ & $6.5(10.2) ; n=79$ & $4.1(5.4) ; n=24$ \\
\hline SD of inorganic & $2.1(3.5) ; n=113$ & $0.5(0.7) ; n=13$ & $2.3(3.7) ; n=100$ & $0.7^{*}$ & $0.8(1.4) ; n=41$ & $0.5(0.7) ; n=13$ \\
\hline \multicolumn{7}{|l|}{$\begin{array}{l}\text { Glycemic and } \\
\text { clotting profile }\end{array}$} \\
\hline $\begin{array}{l}\text { Triglyceride, } \\
\mathrm{mmol} / \mathrm{L}\end{array}$ & $1.4(1.1) ; n=598$ & $1.5(1.0) ; n=68$ & $1.4(1.1) ; n=530$ & 0.08 & $1.5(1.2) ; n=214$ & $1.5(1.05) ; \mathrm{n}=68$ \\
\hline SD of triglyceride & $0.4(0.5) ; n=294$ & $0.4(0.43) ; \mathrm{n}=30$ & $0.35(0.54) ; \mathrm{n}=264$ & 0.1 & $0.4(0.6) ; n=90$ & $0.4(0.43) ; n=30$ \\
\hline Glucose, mmol/L & $6.3(2.3) ; n=1773$ & $6.4(2.7) ; n=206$ & $6.2(2.2) ; n=1567$ & 0.09 & $6.3(2.2) ; n=608$ & $6.4(2.7) ; n=206$ \\
\hline SD of glucose & $1.5(1.3) ; n=1638$ & 1.4(1.3);n = 194 & $1.5(1.3) ; \mathrm{n}=1444$ & 0.09 & $1.5(1.4) ; n=564$ & $1.4(1.3) ; n=194$ \\
\hline $\mathrm{HbA} 1 \mathrm{c}, \mathrm{g} / \mathrm{dL}$ & $12.8(2.0) ; \mathrm{n}=1953$ & $13.3(1.7) ; n=221$ & $12.8(2.0) ; n=1732$ & $0.31 *$ & $13.1(1.9) ; n=663$ & $13.3(1.7) ; n=221$ \\
\hline SD of $\mathrm{HbA} 1 \mathrm{c}$ & $1.3(0.5) ; n=1947$ & $1.31(0.54) ; n=220$ & $1.29(0.54) ; n=1727$ & 0.05 & $1.3(0.5) ; n=660$ & $1.31(0.54) ; n=220$ \\
\hline $\begin{array}{l}\text { High sensitive } \\
\text { troponin-I, ng/L }\end{array}$ & $\begin{array}{l}363.3(10967.7) ; \mathrm{n}= \\
1141\end{array}$ & $12.7(31.8) ; \mathrm{n}=122$ & $\begin{array}{l}405.3(11605.6) ; \mathrm{n}= \\
1019\end{array}$ & 0.05 & $35.5(314.8) ; n=372$ & $12.7(31.8) ; n=122$ \\
\hline $\begin{array}{l}\text { SD of high } \\
\text { sensitive troponin- } \\
\text { I }\end{array}$ & $124.0(1328.2) ; \mathrm{n}=755$ & $18.2(42.4) ; n=82$ & $136.9(1406.3) ; n=673$ & 0.12 & $34.7(207.0) ; n=245$ & $18.2(42.4) ; n=82$ \\
\hline APTT, second & $30.8(5.5) ; n=694$ & $29.1(3.5) ; n=46$ & $30.9(5.6) ; n=648$ & $0.39 *$ & $30.4(3.9) ; n=217$ & $29.1(3.5) ; n=46$ \\
\hline SD of APTT & $2.6(3.1) ; n=510$ & $1.9(2.3) ; n=26$ & $2.6(3.2) ; n=484$ & $0.28^{*}$ & $2.1(1.8) ; n=152$ & $1.9(2.3) ; n=26$ \\
\hline $\begin{array}{l}\text { Lactate } \\
\text { dehydrogenase, } \\
\text { U/L }\end{array}$ & $324.1(422.0) ; n=1431$ & $315.6(534.6) ; n=145$ & $325.1(407.6) ; n=1286$ & 0.02 & $302.1(354.0) ; \mathrm{n}=466$ & $315.6(534.6) ; n=145$ \\
\hline $\begin{array}{l}\text { SD of lactate } \\
\text { dehydrogenase }\end{array}$ & $155.2(397.1) ; \mathrm{n}=1140$ & 115.6(203.9);n = 102 & $159.1(411.1) ; \mathrm{n}=1038$ & 0.13 & $121.3(206.3) ; \mathrm{n}=365$ & $115.6(203.9) ; n=102$ \\
\hline $\begin{array}{l}\text { Total cholesterol, } \\
\mathrm{mmol} / \mathrm{L}\end{array}$ & $4.6(1.1) ; n=599$ & $4.9(1.0) ; n=68$ & $4.5(1.1) ; n=531$ & $0.29 *$ & $4.6(1.1) ; n=214$ & $4.9(1.0) ; n=68$ \\
\hline $\begin{array}{l}\text { SD of total } \\
\text { cholesterol }\end{array}$ & $0.5(0.4) ; n=295$ & $0.54(0.43) ; n=31$ & $0.48(0.44) ; n=264$ & 0.14 & $0.5(0.5) ; n=93$ & $0.54(0.43) ; n=31$ \\
\hline $\begin{array}{l}\text { Low-density } \\
\text { lipoprotein, } \\
\text { mmol/L }\end{array}$ & $2.6(0.9) ; n=583$ & $2.9(0.9) ; n=65$ & $2.6(0.9) ; n=518$ & $0.34^{\star}$ & $2.7(1.0) ; n=208$ & $2.9(0.9) ; n=65$ \\
\hline $\begin{array}{l}\text { SD of low-density } \\
\text { lipoprotein }\end{array}$ & $0.4(0.4) ; n=283$ & $0.5(0.4) ; n=29$ & $0.4(0.4) ; n=254$ & 0.18 & $0.4(0.4) ; n=88$ & $0.5(0.4) ; n=29$ \\
\hline $\begin{array}{l}\text { High-density } \\
\text { lipoprotein, } \\
\text { mmol/L }\end{array}$ & $1.3(0.4) ; n=593$ & $1.32(0.4) ; \mathrm{n}=67$ & $1.34(0.4) ; n=526$ & 0.04 & $1.3(0.4) ; n=212$ & $1.32(0.4) ; n=67$ \\
\hline $\begin{array}{l}\text { SD of high-density } \\
\text { lipoprotein }\end{array}$ & $0.1(0.1) ; n=280$ & $0.12(0.09) ; n=30$ & $0.14(0.14) ; \mathrm{n}=250$ & 0.17 & $0.2(0.1) ; n=87$ & $0.1(0.1) ; n=30$ \\
\hline
\end{tabular}


Table 3

Significant univariate predictors of new onset cardiac complication outcome and all-cause mortality before and after 1:2 propensity score matching

Characteristics

Before matching

Composite outcome $(\mathrm{N}$ $=320)$ Mean(SD); $\mathrm{N}$ or Count(\%)

Demographics

Male gender

$219(68.43 \%)$

$1122(68.45 \%)$

Female gender

$101(31.56 \%)$

$517(31.54 \%)$

$64.0(12.8) ; n=320$

$16(5.00 \%)$

$<40$

$[40,50)$

$[50-60)$

$24(7.50 \%)$

$70(21.87 \%)$

[60-70)

[70-80)

$>=80$

$106(33.12 \%)$

$74(23.12 \%)$

$30(9.37 \%)$

No composite outcome ( = 1639) Mean(SD); $\mathrm{N}$ or Count(\%)

SMD

After 1:2 matching

SMD $(\mathrm{N}=91)$ Mean(SD); $\mathrm{N}$ or Count(\%)

No composite outcome $(\mathrm{N}=572$ Mean(SD); $\mathrm{N}$ or Count(\%)

Past comorbidities

Charlson standard comorbidity index

Hypertension

6.7(3.3);n = 320

$6.0(3.3) ; n=1639$

$0.23 * \quad 7.0(3.1) ; \mathrm{n}=91$

6.4(3.1); $\mathrm{n}=572$

0.18

Liver diseases

$49(15.31 \%)$

$22(6.87 \%)$

207(12.62\%)

$171(10.43 \%)$

$0.08 \quad 13(14.28 \%)$

$58(3.53 \%)$

$0.133(3.29 \%)$

Hip fractures/accident $\quad 19(5.93 \%)$

falls

Renal diseases

Diabetes mellitus

$55(17.18 \%)$

Maligt dysrhythmia

$30(9.37 \%)$

237(14.46\%)

$126(7.68 \%)$

$2(0.12 \%)$

Chronic obstructiv

pulmonary disease

Ischemic heart disease

$10(3.12 \%)$

$3(0.93 \%)$

12(0.73\%)

19(5.93\%)

$42(2.56 \%)$

3(0.93\%)

$8(0.48 \%)$

disease

Endocrine diseases

76(23.75\%)

$472(28.79 \%)$

$1182(72.11 \%)$

63(3.84\%)

$17(5.31 \%)$

Stroke/transient

ischemic attack

Hospitalization

Average readmission

45.2(87.1); $\mathrm{n}=312$

73.1(209.5); $\mathrm{n}=1564$

14.4(14.4); $\mathrm{n}=1564$

13.2(11.4); $\mathrm{n}=312$

40.4(38.3); $n=312$

Overall hospital stay, days

Medications

PD-L1 v.s. PD-1

PD-L1 expenditure,

HKD

Total PD-L1 dose amount, $\mathrm{mg}$

$11(3.43 \%)$
$58752.6(47219.8) ; n=11$
$5127.3(4650.4) ; n=11$

100319.9(100303.8); $\mathrm{n}=\quad$ 0.53* 58752.6(47219.8); $\mathrm{n}=$ 210

$13037.9(28128.8) ; n=210$ 11
$0.1731 .0(35.6) ; \mathrm{n}=90$

$0.119(9.89 \%)$

$0.07 \quad 12(13.18 \%)$

$0.06 \quad 10(10.98 \%)$

$0.24^{*} \quad 0(0.00 \%)$

$0.02 \quad 2(2.19 \%)$

$0.173(3.29 \%)$

$0.1120(21.97 \%)$

$0.01 \quad 82(90.10 \%)$

$0.07 \quad 3(3.29 \%)$

$0.09 \quad 14.5(9.0) ; \mathrm{n}=90$

$0.16 \quad 44.7(34.7) ; \mathrm{n}=90$

$0.35^{\star}$
$0.53^{\star}$
$0.39 *$

$429(75.00 \%)$

$143(25.00 \%)$

0.02

63.1(10.1); $\mathrm{n}=572 \quad 0.04$

$16(2.79 \%)$

0.09

$37(6.46 \%)$

0.01

131(22.90\%)

0.08

252(44.05\%)

0.09

$116(20.27 \%)$

0.04

$20(3.49 \%)$

0.05

34.2(38.1); $\mathrm{n}=1564$

$75(13.11 \%)$

0.03

$21(3.67 \%)$

0.02

$28(4.89 \%)$

0.19

73(12.76\%)

0.01

$50(8.74 \%)$

0.08

$2(0.34 \%)$

0.08

$4(0.69 \%)$

0.13

$12(2.09 \%)$

0.07

$2(0.34 \%)$

0.09

$146(25.52 \%)$

0.08

$469(81.99 \%)$

0.24 *

$21(3.67 \%)$

0.02

* for $p \leq 0.05$, ** for $p \leq 0.01$, *** for $p \leq 0.001$; APTT: applied partial thromboplastin test; PD-1: Programmed death 1 inhibitors; PD-L1: programmed death 1 ligand inhibitors; Cl: Confidence interval; HR: Hazard ratio.

$\begin{array}{ll}210(36.71 \%) & 0.60 * \\ 100319.9(100303.8) ; n= & 0.53^{*} \\ 210 & \\ 13037.9(28128.8) ; n= & 0.39 * \\ 210 & \end{array}$




\begin{tabular}{|c|c|c|c|c|c|c|}
\hline \multirow[t]{2}{*}{ Characteristics } & \multicolumn{2}{|l|}{ Before matching } & \multirow[t]{2}{*}{ SMD } & \multicolumn{2}{|l|}{ After $1: 2$ matching } & \multirow[t]{2}{*}{ SMD } \\
\hline & $\begin{array}{l}\text { Composite outcome }(\mathrm{N} \\
=320) \text { Mean(SD); } \mathrm{N} \text { or } \\
\text { Count(\%) }\end{array}$ & $\begin{array}{l}\text { No composite outcome ( } \mathrm{N} \\
=1639) \text { Mean(SD);N or } \\
\text { Count(\%) }\end{array}$ & & $\begin{array}{l}\text { Composite outcome } \\
(\mathrm{N}=91) \text { Mean(SD);N or } \\
\text { Count(\%) }\end{array}$ & $\begin{array}{l}\text { No composite outcome } \\
(\mathrm{N}=572) \text { Mean(SD);N or } \\
\text { Count(\%) }\end{array}$ & \\
\hline $\begin{array}{l}\text { PD-L1 inhibitors } \\
\text { duration, days }\end{array}$ & $84.6(114.7) ; n=11$ & $180.7(203.5) ; n=210$ & $0.58^{*}$ & $84.6(114.7) ; n=11$ & $180.7(203.5) ; \mathrm{n}=210$ & $0.58^{*}$ \\
\hline PD-1 expenditure & $\begin{array}{l}163440.0(284113.0) ; n= \\
309\end{array}$ & $\begin{array}{l}200406.0(293311.2) ; n= \\
1441\end{array}$ & 0.13 & $\begin{array}{l}\text { 183754.8(224766.1);n } \\
=80\end{array}$ & $\begin{array}{l}208066.4(265897.1) ; n= \\
374\end{array}$ & 0.1 \\
\hline $\begin{array}{l}\text { Total PD-1 dose } \\
\text { amount (MG) }\end{array}$ & $5146.2(21240.1) ; \mathrm{n}=309$ & 2318.6(5983.5);n = 1441 & 0.18 & $3948.7(9243.4) ; \mathrm{n}=80$ & $2135.1(3682.3) ; n=374$ & $0.26^{*}$ \\
\hline $\begin{array}{l}\text { PD-1 inhibitors } \\
\text { duration, days }\end{array}$ & 172.1(197.1);n = 309 & 209.3(245.1);n= 1441 & 0.17 & $173.9(197.7) ; \mathrm{n}=80$ & $214.9(241.5) ; \mathrm{n}=374$ & 0.19 \\
\hline Anticoagulants & $171(53.43 \%)$ & $937(57.16 \%)$ & 0.08 & $47(51.64 \%)$ & $315(55.06 \%)$ & 0.07 \\
\hline Steroids & $171(53.43 \%)$ & $937(57.16 \%)$ & 0.08 & $47(51.64 \%)$ & $315(55.06 \%)$ & 0.07 \\
\hline \multicolumn{7}{|l|}{$\begin{array}{l}\text { Inflammatory } \\
\text { subclinical biomarkers }\end{array}$} \\
\hline $\begin{array}{l}\text { Neutrophil-to- } \\
\text { lymphocyte ratio }\end{array}$ & $4.3(6.8) ; n=320$ & $4.6(6.3) ; n=1632$ & 0.05 & $3.5(1.8) ; n=91$ & $4.4(6.9) ; n=572$ & 0.19 \\
\hline $\begin{array}{l}\text { Platelet-to-lymphocyte } \\
\text { ratio }\end{array}$ & $209.3(237.5) ; \mathrm{n}=320$ & $212.9(245.2) ; n=1633$ & 0.02 & 199.9(135.5);n = 91 & $211.8(323.2) ; n=572$ & 0.05 \\
\hline $\begin{array}{l}\text { Aspartate } \\
\text { transaminase-to- } \\
\text { alanine transaminase } \\
\text { ratio }\end{array}$ & $1.7(1.7) ; n=204$ & $1.8(3.9) ; n=1104$ & 0.05 & $1.2(0.6) ; \mathrm{n}=52$ & $1.5(2.2) ; n=353$ & 0.2 \\
\hline $\begin{array}{l}\text { Triglyceride glucose } \\
\text { index }\end{array}$ & $6.9(0.5) ; n=106$ & $7.1(0.6) ; n=474$ & $0.35^{\star}$ & $6.8(0.5) ; n=28$ & $7.1(0.6) ; n=179$ & $0.44^{*}$ \\
\hline Urea-to-creatinine ratio & $72.9(30.7) ; n=317$ & $73.2(42.5) ; n=1620$ & 0.01 & $78.0(45.9) ; \mathrm{n}=91$ & $68.1(25.3) ; n=568$ & $0.26^{*}$ \\
\hline $\begin{array}{l}\text { Monocyte-to- } \\
\text { lymphocyte ratio }\end{array}$ & $0.4(0.4) ; n=319$ & $0.5(0.5) ; \mathrm{n}=1631$ & 0.05 & $0.44(0.36) ; n=90$ & $0.45(0.45) ; n=572$ & 0.03 \\
\hline \multicolumn{7}{|l|}{ Complete blood counts } \\
\hline $\begin{array}{l}\text { Mean corpuscular } \\
\text { volume, } \mathrm{fL}\end{array}$ & $88.9(7.8) ; n=320$ & $87.8(8.2) ; n=1633$ & 0.13 & $89.9(5.1) ; n=91$ & $87.9(8.0) ; n=572$ & $0.29 *$ \\
\hline Eosinophil, $x 10^{\wedge} 9 / \mathrm{L}$ & $0.18(0.2) ; n=320$ & $0.19(0.33) ; n=1632$ & 0.02 & $0.21(0.21) ; \mathrm{n}=91$ & $0.2(0.27) ; \mathrm{n}=572$ & 0.05 \\
\hline Lymphocyte, $\times 10^{\wedge} 9 / \mathrm{L}$ & $1.6(1.0) ; n=320$ & $1.5(0.9) ; n=1633$ & 0.08 & $1.6(0.6) ; \mathrm{n}=91$ & $1.5(0.7) ; n=572$ & 0.09 \\
\hline $\begin{array}{l}\text { Metamyelocyte, } \\
\text { x10^9/L }\end{array}$ & $0.3(0.6) ; n=37$ & $0.9(5.5) ; n=188$ & 0.13 & $0.35(0.36) ; \mathrm{n}=11$ & $0.34(0.53) ; n=61$ & 0.02 \\
\hline Monocyte, $\times 10^{\wedge} 9 / \mathrm{L}$ & $0.54(0.3) ; n=320$ & $0.53(0.31) ; n=1633$ & 0.02 & $0.6(0.4) ; n=91$ & $0.5(0.3) ; n=572$ & 0.1 \\
\hline Neutrophil, x10^9/L & $5.0(3.2) ; n=320$ & $5.1(3.3) ; n=1633$ & 0.02 & $4.99(2.76) ; n=91$ & $5.02(3.28) ; n=572$ & 0.01 \\
\hline $\begin{array}{l}\text { White blood count, } \\
\times 10^{\wedge} 9 / \mathrm{L}\end{array}$ & $7.41(3.71) ; n=320$ & $7.39(5.8) ; n=1633$ & 0 & $7.4(3.2) ; n=91$ & 7.3(3.7);n = 572 & 0.01 \\
\hline $\begin{array}{l}\text { Mean cell } \\
\text { haemoglobin, pg }\end{array}$ & $30.9(3.2) ; n=320$ & $30.6(3.4) ; n=1633$ & 0.08 & $31.4(2.4) ; \mathrm{n}=91$ & $30.7(3.3) ; n=572$ & $0.24 *$ \\
\hline Myelocyte, $\times 10^{\wedge} 9 / \mathrm{L}$ & $0.5(0.9) ; \mathrm{n}=50$ & $0.8(3.3) ; n=278$ & 0.14 & $0.4(0.5) ; n=16$ & $0.7(1.6) ; n=81$ & $0.27 *$ \\
\hline Platelet, $\times 10^{\wedge} 9 / \mathrm{L}$ & $248.7(100.8) ; n=320$ & $247.8(110.0) ; n=1633$ & 0.01 & 258.9(81.8);n = 91 & 251.6(101.4);n = 572 & 0.08 \\
\hline $\begin{array}{l}\text { Red blood count, } \\
\text { x10^12/L }\end{array}$ & $4.36(0.68) ; n=320$ & $4.4(0.71) ; n=1633$ & 0.06 & $4.54(0.51) ; n=91$ & $4.45(0.68) ; n=572$ & 0.14 \\
\hline Hematocrit, L/L & $0.38(0.05) ; n=303$ & $0.38(0.06) ; n=1592$ & 0.02 & $0.41(0.04) ; n=79$ & $0.39(0.05) ; \mathrm{n}=547$ & $0.39 *$ \\
\hline \multicolumn{7}{|l|}{$\begin{array}{l}\text { Renal and liver } \\
\text { functions }\end{array}$} \\
\hline $\mathrm{K} /$ Potassium, mmol/L & $4.14(0.45) ; n=318$ & $4.12(0.43) ; n=1623$ & 0.03 & $4.12(0.39) ; \mathrm{n}=91$ & $4.13(0.43) ; n=570$ & 0.02 \\
\hline Urate, mmol/L & $0.34(0.17) ; n=96$ & $0.32(0.13) ; n=531$ & 0.14 & $0.33(0.19) ; n=32$ & $0.34(0.11) ; \mathrm{n}=154$ & 0.02 \\
\hline Albumin, $\mathrm{g} / \mathrm{L}$ & $39.17(5.99) ; n=318$ & $39.2(5.89) ; n=1621$ & 0 & $40.4(4.3) ; n=91$ & $39.4(5.6) ; n=569$ & $0.21^{*}$ \\
\hline
\end{tabular}

* for $\mathrm{p} \leq 0.05$, ** for $\mathrm{p} \leq 0.01, * * *$ for $\mathrm{p} \leq 0.001$; APTT: applied partial thromboplastin test; PD-1: Programmed death 1 inhibitors; PD-L1: programmed death 1 ligand inhibitors; Cl: Confidence interval; HR: Hazard ratio. 


\begin{tabular}{|c|c|c|c|c|c|c|}
\hline \multirow[t]{2}{*}{ Characteristics } & \multicolumn{2}{|l|}{ Before matching } & \multirow[t]{2}{*}{ SMD } & \multicolumn{2}{|l|}{ After 1:2 matching } & \multirow[t]{2}{*}{ SMD } \\
\hline & $\begin{array}{l}\text { Composite outcome ( } \mathrm{N} \\
=320) \text { Mean(SD); } \mathrm{N} \text { or } \\
\text { Count(\%) }\end{array}$ & $\begin{array}{l}\text { No composite outcome ( } \mathrm{N} \\
=1639) \text { Mean(SD);N or } \\
\text { Count(\%) }\end{array}$ & & $\begin{array}{l}\text { Composite outcome } \\
(\mathrm{N}=91) \text { Mean(SD);N or } \\
\text { Count(\%) }\end{array}$ & $\begin{array}{l}\text { No composite outcome } \\
(\mathrm{N}=572) \text { Mean(SD); } \mathrm{N} \text { or } \\
\text { Count(\%) }\end{array}$ & \\
\hline $\mathrm{Na} /$ Sodium, mmol/L & $139.0(3.8) ; n=318$ & $139.1(3.8) ; n=1623$ & 0.03 & 139.2(3.6);n = 91 & 139.3(3.4);n = 570 & 0.01 \\
\hline Urea, mmol/L & $5.7(2.8) ; \mathrm{n}=317$ & $5.5(2.4) ; n=1620$ & 0.08 & $5.34(2.09) ; \mathrm{n}=91$ & $5.3(1.76) ; n=568$ & 0.02 \\
\hline Protein, $\mathrm{g} / \mathrm{L}$ & $72.6(12.5) ; \mathrm{n}=308$ & $72.0(12.9) ; n=1545$ & 0.04 & $72.2(12.5) ; \mathrm{n}=87$ & $71.6(14.7) ; n=537$ & 0.05 \\
\hline Bilirubin, umol/L & $11.9(16.6) ; n=318$ & $13.2(26.6) ; n=1623$ & 0.05 & $10.9(7.3) ; \mathrm{n}=91$ & $11.4(20.3) ; \mathrm{n}=570$ & 0.04 \\
\hline Creatinine, umol/L & $84.6(44.3) ; n=320$ & $85.0(65.3) ; n=1632$ & 0.01 & $78.0(42.3) ; n=91$ & $85.9(66.8) ; n=571$ & 0.14 \\
\hline SD of creatinine & $39.6(128.3) ; n=319$ & $34.7(98.1) ; n=1623$ & 0.04 & 28.8(55.2);n = 91 & 29.9(85.9);n = 567 & 0.01 \\
\hline $\begin{array}{l}\text { Aspartate } \\
\text { transaminase, U/L }\end{array}$ & $45.2(56.6) ; n=221$ & $56.2(111.7) ; n=1150$ & 0.12 & $29.0(15.0) ; n=64$ & $43.3(72.3) ; n=379$ & $0.27 *$ \\
\hline $\begin{array}{l}\text { SD of aspartate } \\
\text { transaminase }\end{array}$ & 57.8(157.0);n = 198 & $59.9(195.2) ; n=1052$ & 0.01 & $30.5(74.7) ; n=62$ & $37.5(85.9) ; n=347$ & 0.09 \\
\hline $\begin{array}{l}\text { Alkaline phosphatase, } \\
\text { U/L }\end{array}$ & $98.9(76.8) ; n=318$ & $110.5(117.1) ; n=1623$ & 0.12 & 87.6(42.5);n = 91 & 104.4(106.1);n = 570 & $0.21^{*}$ \\
\hline $\begin{array}{l}\text { SD of alkaline } \\
\text { phosphatase }\end{array}$ & $68.8(96.7) ; n=317$ & $64.0(94.8) ; n=1611$ & 0.05 & $62.9(90.6) ; n=90$ & $57.5(89.9) ; \mathrm{n}=565$ & 0.06 \\
\hline $\begin{array}{l}\text { Alanine transaminase, } \\
\mathrm{U} / \mathrm{L}\end{array}$ & $36.0(69.2) ; \mathrm{n}=301$ & $36.7(52.8) ; n=1575$ & 0.01 & 26.7(16.3);n = 79 & $32.1(30.9) ; n=544$ & $0.22^{*}$ \\
\hline $\begin{array}{l}\text { SD of alanine } \\
\text { transaminase }\end{array}$ & $40.6(122.4) ; n=300$ & $35.6(75.3) ; n=1555$ & 0.05 & $21.6(21.1) ; \mathrm{n}=78$ & $29.2(54.6) ; n=534$ & 0.18 \\
\hline \multicolumn{7}{|l|}{$\begin{array}{l}\text { Lipid, iron and calcium } \\
\text { profile }\end{array}$} \\
\hline $\begin{array}{l}\text { Total iron-binding } \\
\text { capacity, L }\end{array}$ & $41.4(14.2) ; n=13$ & $40.9(12.1) ; \mathrm{n}=90$ & 0.04 & $61.3(15.3) ; n=2$ & $40.2(11.1) ; n=20$ & $1.58^{\star}$ \\
\hline VitaminB12, pmol/L & $305.1(109.5) ; \mathrm{n}=6$ & $473.9(342.5) ; n=43$ & $0.66^{*}$ & $304.0(145.7) ; \mathrm{n}=2$ & $414.1(322.1) ; n=15$ & $0.44^{*}$ \\
\hline Folate, $\mathrm{ng} / \mathrm{mL}$ & $26.5(14.2) ; \mathrm{n}=8$ & $21.1(9.5) ; n=61$ & $0.44^{*}$ & $32.3(29.6) ; n=2$ & $19.0(6.8) ; \mathrm{n}=17$ & $0.62^{*}$ \\
\hline Ferritin, pmol/L & $3257.7(4708.7) ; n=12$ & $2431.4(4767.6) ; n=74$ & 0.17 & $5116.7(6053.8) ; n=2$ & $1623.6(2601.0) ; n=20$ & $0.75^{\star}$ \\
\hline Calcium, mmol/L & $2.31(0.14) ; n=185$ & $2.32(0.16) ; n=896$ & 0.06 & $2.34(0.1) ; n=53$ & $2.32(0.15) ; n=287$ & 0.1 \\
\hline SD of calcium & $0.09(0.06) ; \mathrm{n}=167$ & $0.1(0.06) ; n=820$ & 0.05 & $0.09(0.06) ; n=48$ & $0.09(0.07) ; \mathrm{n}=266$ & 0.01 \\
\hline Phosphate, mmol/L & $1.0(0.2) ; n=159$ & $1.1(0.2) ; n=789$ & 0.05 & $1.0(0.2) ; n=41$ & $1.1(0.2) ; n=245$ & 0.09 \\
\hline SD of phosphate & $0.17(0.12) ; n=122$ & $0.16(0.11) ; \mathrm{n}=663$ & 0.05 & $0.17(0.12) ; n=33$ & $0.15(0.09) ; \mathrm{n}=208$ & 0.16 \\
\hline Inorganic, mmol/L & $4.8(5.7) ; n=36$ & $6.7(9.0) ; n=223$ & $0.24^{*}$ & $3.5(5.4) ; n=8$ & $6.9(10.5) ; n=71$ & $0.41^{\star}$ \\
\hline SD of inorganic & $2.4(3.8) ; n=17$ & $2.1(3.5) ; n=96$ & 0.1 & $0.2(0.1) ; \mathrm{n}=6$ & $0.9(1.5) ; n=35$ & $0.69 *$ \\
\hline \multicolumn{7}{|l|}{$\begin{array}{l}\text { Glycemic and clotting } \\
\text { profile }\end{array}$} \\
\hline Triglyceride, $\mathrm{mmol} / \mathrm{L}$ & $1.2(0.8) ; \mathrm{n}=110$ & $1.5(1.1) ; \mathrm{n}=488$ & $0.24^{*}$ & $1.1(0.7) ; n=30$ & $1.5(1.3) ; \mathrm{n}=184$ & $0.38^{*}$ \\
\hline SD of triglyceride & $0.3(0.4) ; n=46$ & $0.4(0.5) ; n=248$ & $0.23^{\star}$ & $0.3(0.2) ; \mathrm{n}=8$ & $0.4(0.6) ; n=82$ & $0.28^{*}$ \\
\hline Glucose, mmol/L & $6.2(2.1) ; n=294$ & $6.3(2.3) ; n=1479$ & 0.03 & $6.1(1.5) ; n=86$ & $6.3(2.3) ; n=522$ & 0.13 \\
\hline SD of glucose & $1.55(1.26) ; \mathrm{n}=276$ & $1.48(1.3) ; n=1362$ & 0.05 & $1.6(1.4) ; n=83$ & $1.5(1.4) ; \mathrm{n}=481$ & 0.1 \\
\hline $\mathrm{HbA1c}, \mathrm{g} / \mathrm{dL}$ & $12.9(1.9) ; n=320$ & $12.8(2.0) ; n=1633$ & 0.03 & $13.6(1.5) ; \mathrm{n}=91$ & $13.0(1.9) ; n=572$ & $0.35^{\star}$ \\
\hline SD of $\mathrm{HbA} 1 \mathrm{c}$ & $1.4(0.5) ; n=320$ & $1.3(0.6) ; n=1627$ & 0.14 & $1.5(0.4) ; \mathrm{n}=91$ & $1.3(0.5) ; n=569$ & $0.41^{\star}$ \\
\hline $\begin{array}{l}\text { High sensitive } \\
\text { troponin-I, ng/L }\end{array}$ & $1660.7(24305.4) ; n=232$ & $32.2(442.8) ; n=909$ & 0.09 & $106.2(718.1) ; n=70$ & $19.1(50.2) ; \mathrm{n}=302$ & 0.17 \\
\hline $\begin{array}{l}\text { SD of high sensitive } \\
\text { troponin-I }\end{array}$ & $367.5(2445.0) ; n=164$ & $56.5(762.9) ; \mathrm{n}=591$ & 0.17 & $101.3(453.4) ; n=48$ & 18.5(51.9); $\mathrm{n}=197$ & $0.26^{*}$ \\
\hline APTT, second & $30.4(4.2) ; n=118$ & $30.9(5.8) ; n=576$ & 0.09 & $30.36(3.19) ; n=34$ & $30.44(3.98) ; n=183$ & 0.02 \\
\hline
\end{tabular}




\begin{tabular}{|c|c|c|c|c|c|c|}
\hline \multirow[t]{2}{*}{ Characteristics } & \multicolumn{2}{|l|}{ Before matching } & \multirow[t]{2}{*}{ SMD } & \multicolumn{2}{|l|}{ After 1:2 matching } & \multirow[t]{2}{*}{ SMD } \\
\hline & $\begin{array}{l}\text { Composite outcome ( } \mathrm{N} \\
=320) \text { Mean(SD); } \mathrm{N} \text { or } \\
\text { Count(\%) }\end{array}$ & $\begin{array}{l}\text { No composite outcome ( } \mathrm{N} \\
=1639) \text { Mean(SD); } \mathrm{N} \text { or } \\
\text { Count(\%) }\end{array}$ & & $\begin{array}{l}\text { Composite outcome } \\
(\mathrm{N}=91) \text { Mean(SD); } \mathrm{N} \text { or } \\
\text { Count(\%) }\end{array}$ & $\begin{array}{l}\text { No composite outcome } \\
(\mathrm{N}=572) \text { Mean(SD); } \mathrm{N} \text { or } \\
\text { Count(\%) }\end{array}$ & \\
\hline SD of APTT & $2.1(1.6) ; n=79$ & $2.7(3.3) ; n=431$ & $0.24^{\star}$ & $2.0(1.7) ; n=22$ & $2.1(1.8) ; n=130$ & 0.02 \\
\hline $\begin{array}{l}\text { Lactate } \\
\text { dehydrogenase, U/L }\end{array}$ & $326.8(437.7) ; n=247$ & $323.6(418.8) ; n=1184$ & 0.01 & $273.8(128.9) ; n=72$ & $307.3(380.9) ; n=394$ & 0.12 \\
\hline $\begin{array}{l}\text { SD of lactate } \\
\text { dehydrogenase }\end{array}$ & $133.2(228.0) ; n=182$ & $159.3(421.6) ; n=958$ & 0.08 & $131.0(187.3) ; \mathrm{n}=57$ & $119.5(209.9) ; \mathrm{n}=308$ & 0.06 \\
\hline $\begin{array}{l}\text { Total cholesterol, } \\
\mathrm{mmol} / \mathrm{L}\end{array}$ & $4.4(1.0) ; n=110$ & $4.6(1.1) ; n=489$ & 0.18 & $4.9(1.4) ; n=30$ & $4.6(1.0) ; n=184$ & $0.24^{*}$ \\
\hline SD of total cholesterol & $0.4(0.4) ; n=46$ & $0.5(0.4) ; n=249$ & 0.15 & $0.7(0.9) ; \mathrm{n}=8$ & $0.5(0.4) ; n=85$ & $0.32^{*}$ \\
\hline $\begin{array}{l}\text { Low-density } \\
\text { lipoprotein, } \mathrm{mmol} / \mathrm{L}\end{array}$ & $2.5(0.9) ; n=109$ & $2.6(0.9) ; n=474$ & 0.13 & $3.0(1.3) ; n=30$ & $2.6(0.9) ; n=178$ & $0.3^{*}$ \\
\hline $\begin{array}{l}\text { SD of low-density } \\
\text { lipoprotein }\end{array}$ & $0.38(0.35) ; n=44$ & $0.42(0.37) ; n=239$ & 0.11 & $0.7(0.8) ; \mathrm{n}=8$ & $0.4(0.3) ; n=80$ & $0.45^{*}$ \\
\hline $\begin{array}{l}\text { High-density } \\
\text { lipoprotein, } \mathrm{mmol} / \mathrm{L}\end{array}$ & $1.4(0.4) ; n=109$ & $1.3(0.4) ; n=484$ & 0.07 & $1.4(0.3) ; n=30$ & $1.3(0.4) ; n=182$ & $0.26^{*}$ \\
\hline $\begin{array}{l}\text { SD of high-density } \\
\text { lipoprotein }\end{array}$ & $0.14(0.13) ; n=42$ & $0.14(0.13) ; n=238$ & 0.02 & $0.1(0.1) ; n=8$ & $0.2(0.1) ; n=79$ & 0.02 \\
\hline
\end{tabular}

Univariate Cox regression identified significant predictors of the primary composite outcome and all-cause mortality before and after propensity score matching (Table 3). Compared with PD-1 inhibitor treatment, PD-L1 inhibitor treatment was significantly associated with a lower risk of composite outcome both before (hazard ratio [HR]: $0.32,95 \% \mathrm{Cl}$ : [0.18-0.59], P value $\left.=0.0002^{\star \star *}\right)$ and after matching $(\mathrm{HR}: 0.34,95 \% \mathrm{Cl}:[0.18-0.65], \mathrm{P}$ value $=0.001 * \star)$, and lower all-cause mortality risk before matching (HR: $0.77,95 \% \mathrm{Cl}$ : [0.64-0.93], $\mathrm{P}$ value $\left.=0.0078^{\star \star}\right)$ and after matching $(\mathrm{HR}: 0.80,95 \% \mathrm{Cl}:[0.65-1.00], \mathrm{P}$ value $=0.0463)$.

More PD-L1 expenditure (HR:1.000; 95\% Cl: 1.000-1.000; P value = 0.0528), shorter PD-L1 inhibitors duration (HR:0.99; 95\% Cl: 0.99-1.00; P value = 0.0263*), more PD-1 expenditure (HR:1.000; 95\% Cl: 1.000-1.001; $\mathrm{P}$ value $\left.=0.0018^{\star *}\right)$, and shorter PD-1 inhibitors duration (HR:0.998; 95\% Cl: 0.997-0.999; P value = $\left.0.0007^{\star \star *}\right)$ were associated with new onset cardiac composite outcome in the matched cohort. Significant laboratory examinations significantly associated with new onset cardiac composite outcome include higher levels of mean corpuscular volume (HR:1.03; 95\% $\mathrm{Cl}: 1.00-1.07 ; \mathrm{P}$ value $=0.0470 *$ ), hematocrit (HR:566.25; 95\% Cl: 4.88-65751.46; P value = 0.0090**), HbA1c (HR:1.19; 95\% Cl: 1.05-1.34; P value = 0.0072**), total cholesterol (HR:1.42; 95\% Cl: 1.00-2.00; $\mathrm{P}$ value $\left.=0.0490^{\star}\right)$, and low-density lipoprotein (HR:1.67; 95\% Cl: $1.14-2.45 ; \mathrm{P}$ value $\left.=0.0091^{\star \star}\right)$.

In addition, greater variability in laboratory tests, including the standard deviations (SD) of alkaline phosphatase (HR:1.002; 95\% Cl: 1.000-1.004; P value = 0.0359*), HbA1c (HR:1.94; 95\% Cl: 1.36-2.76; P value = 0.0003***), lactate dehydrogenase (HR:1.002; 95\% Cl: 1.000-1.003; P value = 0.0056**), and lowdensity lipoprotein (HR:7.64; $95 \% \mathrm{Cl}: 1.84-31.77$; P value $=0.0051^{\star \star}$ ) were significantly associated with the composite outcome. The boxplots of significant measures of variability stratified by PD-1/PD-L1 inhibitor treatment in the matched cohort are shown in Fig. 5.

\section{Healthcare utilization before and after treatment with PD-1/PD-L1 inhibitors}

Longer overall cumulative hospital stay (HR:1.01; 95\% Cl: 1.00-1.01; P value $=0.0040 * *)$ and longer hospital stay after PD-1/PD-L1 drug use (HR:1.01; 95\% Cl: 1.00-1.01; P value $=0.0040^{* *}$ ) were significantly associated with the composite outcome. Furthermore, hospitalization characteristics before and after PD1/PD-L1 treatment were compared in the subset of patients who developed the adverse outcomes, in both the unmatched and matched cohorts (Table 4). Patients who developed cardiovascular complications had a shorter average readmission interval, a higher number of hospitalizations and a longer duration of hospital stay after PD-1/PD-L1 treatment $(P<0.0001)$. 
Table 4

Comparisons of hospitalization characteristics before and after PD1/PD-L1 treatment in patients with new onset heart failure, acute myocardial infarction, atrial fibrillation, and atrial flutter

\begin{tabular}{|c|c|c|c|c|}
\hline \multirow[t]{3}{*}{ Characteristics } & \multicolumn{2}{|l|}{ Before matching } & \multicolumn{2}{|l|}{ After 1:2 matching } \\
\hline & All-cause mortality & Composite outcome & All-cause mortality & Composite outcome \\
\hline & HR [95\% Cl];P value & HR [95\% Cl];P value & HR [95\% Cl];P value & HR [95\% Cl];P value \\
\hline \multicolumn{5}{|l|}{ Demographics } \\
\hline Male gender & $0.98[0.87-1.10] ; 0.6878$ & $1.00[0.78-1.29] ; 0.9898$ & $1.03[0.83-1.29] ; 0.7733$ & $1.12[0.67-1.88] ; 0.6546$ \\
\hline Female gender & 1.0[Reference] & 1.0[Reference] & 1.0[Reference] & 1.0[Reference] \\
\hline Baseline age, years & $1.00[1.00-1.01] ; 0.1533$ & $\begin{array}{l}1.01[1.01- \\
1.02] ; 0.0016 \text { ** }\end{array}$ & $1.01[1.00-1.01] ; 0.2976$ & $1.00[0.98-1.02] ; 0.9421$ \\
\hline$<40$ & 1.0[Reference] & 1.0[Reference] & 1.0[Reference] & 1.0[Reference] \\
\hline$[40,50)$ & $1.02[0.85-1.22] ; 0.8400$ & $0.72[0.46-1.12] ; 0.1450$ & $1.07[0.73-1.56] ; 0.7308$ & $0.96[0.39-2.38] ; 0.9321$ \\
\hline$[50-60)$ & $1.04[0.92-1.18] ; 0.5569$ & $0.97[0.74-1.28] ; 0.8386$ & $0.87[0.69-1.09] ; 0.2345$ & $1.20[0.74-1.95] ; 0.4528$ \\
\hline$[60-70)$ & $1.02[0.91-1.14] ; 0.7455$ & $1.05[0.82-1.35] ; 0.6880$ & $0.98[0.81-1.19] ; 0.8684$ & $0.71[0.45-1.11] ; 0.1309$ \\
\hline$[70-80)$ & $0.98[0.85-1.12] ; 0.7150$ & $1.19[0.91-1.57] ; 0.2103$ & $1.16[0.92-1.47] ; 0.2172$ & $1.13[0.66-1.96] ; 0.6528$ \\
\hline$>=80$ & $1.07[0.86-1.35] ; 0.5366$ & $1.40[0.89-2.21] ; 0.1436$ & $1.03[0.63-1.70] ; 0.9074$ & $1.51[0.55-4.13] ; 0.4210$ \\
\hline \multicolumn{5}{|l|}{ Past comorbidities } \\
\hline Charlson standard comorbidity index & $\begin{array}{l}\text { 1.05[1.04-1.07]; } \\
<0.0001^{\star \star \star *}\end{array}$ & $\begin{array}{l}1.08[1.04-1.12] \\
<0.0001^{\star \star \star}\end{array}$ & $\begin{array}{l}1.08[1.04-1.11] \\
<0.0001^{\star \star \star}\end{array}$ & $1.07[1.00-1.15] ; 0.0589$ \\
\hline Hypertension & $1.20[1.02-1.40] ; 0.0262^{*}$ & $1.11[0.78-1.59] ; 0.5645$ & $1.32[1.00-1.73] ; 0.0481^{*}$ & $1.18[0.61-2.29] ; 0.6236$ \\
\hline Liver diseases & $1.24[1.04-1.47] ; 0.0163^{\star}$ & $0.77[0.49-1.22] ; 0.2689$ & $1.46[0.91-2.35] ; 0.1148$ & $1.32[0.42-4.18] ; 0.6381$ \\
\hline Hip fractures/accident falls & $1.26[0.96-1.64] ; 0.0910$ & $\begin{array}{l}1.98[1.21- \\
3.23] ; 0.0065^{\star \star}\end{array}$ & $0.79[0.50-1.25] ; 0.3174$ & $2.21[1.10-4.41] ; 0.0253^{*}$ \\
\hline Renal diseases & $1.02[0.88-1.19] ; 0.7670$ & $1.06[0.77-1.48] ; 0.7155$ & $1.05[0.79-1.39] ; 0.7571$ & $1.24[0.67-2.29] ; 0.4871$ \\
\hline Diabetes mellitus & $1.13[0.92-1.37] ; 0.2369$ & $1.05[0.67-1.66] ; 0.8204$ & $1.12[0.80-1.57] ; 0.4963$ & $1.12[0.52-2.43] ; 0.7733$ \\
\hline Maligt dysrhythmia & $2.09[1.12-3.89] ; 0.0206^{*}$ & $\begin{array}{l}11.11[5.88-20.98] \\
<0.0001_{\star \star \star}\end{array}$ & $0.00[0.00-\operatorname{lnf}] ; 0.9877$ & $0.00[0.00-\operatorname{lnf}] ; 0.9946$ \\
\hline Chronic obstructive pulmonary disease & $1.42[0.78-2.56] ; 0.2515$ & $1.83[0.59-5.72] ; 0.2971$ & $2.04[0.84-4.94] ; 0.1133$ & $\begin{array}{l}5.51[1.34- \\
22.64] ; 0.0179 *\end{array}$ \\
\hline Ischemic heart disease & $0.98[0.72-1.34] ; 0.8984$ & $1.30[0.69-2.44] ; 0.4160$ & $0.98[0.49-1.98] ; 0.9639$ & $0.70[0.10-5.07] ; 0.7271$ \\
\hline Peripheral vascular disease & $1.10[0.57-2.12] ; 0.7774$ & $1.93[0.62-6.03] ; 0.2570$ & $0.87[0.22-3.51] ; 0.8502$ & $2.51[0.35-18.07] ; 0.3611$ \\
\hline Endocrine diseases & $1.04[0.93-1.18] ; 0.4907$ & $0.76[0.58-1.00] ; 0.0538$ & $1.04[0.84-1.29] ; 0.7304$ & $0.75[0.44-1.28] ; 0.2934$ \\
\hline Gastrointestinal diseases & $1.03[0.92-1.17] ; 0.5810$ & $0.94[0.73-1.21] ; 0.6327$ & $1.22[0.93-1.59] ; 0.1534$ & $1.71[0.85-3.41] ; 0.1296$ \\
\hline Stroke/transient ischemic attack & $1.11[0.85-1.46] ; 0.4279$ & $1.02[0.54-1.92] ; 0.9477$ & $1.48[0.91-2.40] ; 0.1149$ & $0.96[0.24-3.92] ; 0.9586$ \\
\hline \multicolumn{5}{|l|}{ Hospitalization } \\
\hline Average readmission & $\begin{array}{l}1.000[1.000- \\
1.000] ; 0.2724\end{array}$ & $\begin{array}{l}0.998[0.997- \\
1.000] ; 0.0625\end{array}$ & $\begin{array}{l}1.000[1.000- \\
1.001] ; 0.0823\end{array}$ & $0.99[0.98-1.00] ; 0.0080 * *$ \\
\hline Total episode number & $\begin{array}{l}0.95[0.95-0.96] \\
<0.0001^{* \star *}\end{array}$ & $\begin{array}{l}0.96[0.95-0.97] \\
<0.0001^{\star \star \star}\end{array}$ & $\begin{array}{l}0.96[0.95-0.97] \\
<0.0001^{\star \star \star}\end{array}$ & $\begin{array}{l}0.97[0.95- \\
0.99] ; 0.0050 * \star\end{array}$ \\
\hline Overall hospital stay, days & $\begin{array}{l}1.001[0.999- \\
1.002] ; 0.3779\end{array}$ & $\begin{array}{l}1.001[0.999- \\
1.004] ; 0.3117\end{array}$ & $\begin{array}{l}1.00[1.00- \\
1.01] ; 0.0004^{\star \star \star}\end{array}$ & $1.01[1.00-1.01] ; 0.0040 * *$ \\
\hline \multicolumn{5}{|l|}{ Medications } \\
\hline PD-L1 v.s. PD-1 & $\begin{array}{l}0.77[0.64- \\
0.93] ; 0.0078^{\star \star}\end{array}$ & $\begin{array}{l}0.32[0.18- \\
0.59] ; 0.0002^{\star \star \star}\end{array}$ & $0.80[0.65-1.00] ; 0.0463^{*}$ & $\begin{array}{l}0.34[0.18- \\
0.65] ; 0.0010^{\star *}\end{array}$ \\
\hline PD-L1 expenditure, HKD & $\begin{array}{l}1.000[1.000-1.000] \\
<0.0001^{* \star *}\end{array}$ & $\begin{array}{l}1.000[1.000- \\
1.000] ; 0.0528\end{array}$ & $\begin{array}{l}1.000[1.000-1.000] \\
<0.0001^{\star \star \star}\end{array}$ & $\begin{array}{l}1.000[1.000- \\
1.000] ; 0.0528\end{array}$ \\
\hline Total PD-L1 dose amount, mg & $\begin{array}{l}1.000[1.000- \\
1.000] ; 0.0703\end{array}$ & $\begin{array}{l}1.000[1.000- \\
1.000] ; 0.2410\end{array}$ & $\begin{array}{l}1.000[1.000- \\
1.000] ; 0.0703\end{array}$ & $\begin{array}{l}1.000[1.000- \\
1.000] ; 0.2410\end{array}$ \\
\hline
\end{tabular}

* for $p \leq 0.05$, ** for $p \leq 0.01$, *** for $p \leq 0.001 ;$ PD-1: Programmed death 1 inhibitors; PD-L1: programmed death 1 ligand inhibitors. 
PD-L1 inhibitors duration, days

PD-1 expenditure

Total PD-1 dose amount (MG)

PD-1 inhibitors duration, days

Anticoagulants

Steroids
1.00[0.99-1.00]; $<0.0001 * * *$

$1.000[1.000-1.000]$; $<0.0001 * * *$

$1.000[1.000-1.000] ;$ $<0.0001^{* * *}$

0.997[0.997-0.998]; $<0.0001 * * *$

$0.81[0.73-$

$0.91] ; 0.0002^{\star * \star}$

$0.81[0.73-$

$0.91] ; 0.0002^{\star \star \star}$
$0.99[0.99-1.00] ; 0.0263^{*}$

$1.000[1.000-1.000]$ $<0.0001^{\star \star \star}$

$1.000[1.000-$ $1.000] ; 0.1842$

0.998[0.997-0.998]; $<0.0001^{\star \star \star}$

$0.73[0.58-$

$0.93] ; 0.0097^{\star *}$

$0.73[0.58-$

$0.93] ; 0.0097 * \star$
1.00[0.99-1.00]; $<0.0001^{\star \star \star}$

$1.000[1.000-1.000]$; $<0.0001^{\star \star *}$

$1.000[1.000-$ $1.000] ; 0.0008^{\star * *}$ $0.998[0.997-0.998]$ $<0.0001^{\star \star *}$

$0.89[0.74-1.08] ; 0.2423$

$0.89[0.74-1.08] ; 0.2423$
0.99[0.99-1.00];0.0263*

$1.000[1.000-$ $1.001] ; 0.0018^{* *}$

$1.000[1.000-$ $1.000] ; 0.0958$

$0.998[0.997-$ $0.999] ; 0.0007 * \star \star$

$0.78[0.50-1.20] ; 0.2574$

$0.78[0.50-1.20] ; 0.2574$

Inflammatory subclinical biomarkers

Neutrophil-to-lymphocyte ratio 1.01[1.00-1.01];0.0296*

1.00[0.98-1.02];0.8231 1.01[1.00-1.02];0.1025

0.97[0.92-1.03];0.3646

Platelet-to-lymphocyte ratio

$1.000[1.000-$

$1.000] ; 0.0261^{*}$

$1.000[1.000-$

$1.001] ; 0.5907$

$1.000[1.000-$

$1.000] ; 0.1087$

$1.000[0.999-$

1.001];0.9257

Aspartate transaminase-to-alanine

1.03[1.01-1.04];

$1.01[0.97-1.06] ; 0.6492$

$1.09[1.04-$

$1.14] ; 0.0004^{\star * \star}$

$0.96[0.71-1.29] ; 0.7683$

Triglyceride glucose index (1)

0.97[0.83-1.13];0.6770

$0.59[0.41-$

$0.85] ; 0.0040$ **

0.85[0.66-1.09];0.2061

$0.55[0.30-1.02] ; 0.0594$

1.000[0.997-

1.002];0.9081

$1.00[1.00-1.01] ; 0.2003$

$1.01[1.00-$

$1.001] ; 0.9657$

0.99[0.79-1.23];0.9303

$1.20[1.02-1.43] ; 0.0305^{\star}$

$1.01] ; 0.0007 * \star \star$

Monocyte-to-lymphocyte ratio

1.07[0.99-1.16];0.0998

1.07[0.69-1.67];0.7559

Complete blood counts

Mean corpuscular volume, $\mathrm{fL}$

Eosinophil, $\times 10^{\wedge} 9 / \mathrm{L}$

1.00[0.99-1.01];0.9942

1.01[1.00-1.03];0.1496

1.00[0.98-1.01];0.5845

1.03[1.00-1.07];0.0470*

Lymphocyte, $x 10^{\wedge} 9 / \mathrm{L}$

0.74[0.58-0.93];0.0105*

0.84[0.54-1.31];0.4333

0.81[0.53-1.23];0.3143

1.14[0.49-2.64];0.7632

0.98[0.92-1.05];0.6221

1.08[0.97-1.20];0.1514

0.96[0.83-1.10];0.5296

1.07[0.78-1.47];0.6699

Metamyelocyte, $\times 10^{\wedge} 9 / \mathrm{L}$

0.87[0.70-1.07];0.1830

$0.78[0.46-1.31] ; 0.3392$

$0.71[0.40-1.28] ; 0.2549$

0.74[0.21-2.63];0.6383

Monocyte, $x 10^{\wedge} 9 / \mathrm{L}$

$1.24[1.06-$

$1.45] ; 0.0078^{\star \star}$

$1.23[0.87-1.73] ; 0.2362 \quad 1.51[1.14-2.00] ; 0.0045^{\star *}$

1.65[0.88-3.11];0.1194

Neutrophil, $\times 10^{\wedge} 9 / \mathrm{L}$

$1.02[1.01-$

$1.04] ; 0.0013^{\star *}$

$1.01[0.98-1.05] ; 0.4025$

$1.03[1.01-1.06] ; 0.0129 *$

$1.02[0.96-1.08] ; 0.5817$

White blood count, $\times 10^{\wedge} 9 / \mathrm{L}$

1.02[1.01-1.03]; $<0.0001^{\star * *}$

Mean cell haemoglobin, pg

0.99[0.97-1.01];0.2267

1.02[1.00-1.05];0.1069

1.03[1.00-1.05];0.0187*

1.02[0.97-1.08];0.4938

0.93[0.84-1.02];0.1131

1.01[0.97-1.05];0.6190

0.96[0.94-0.99];0.0133*

1.05[0.97-1.14];0.1981

Myelocyte, $x 10^{\wedge} 9 / \mathrm{L}$

$1.001[1.000-$

1.001];0.0388*

0.88[0.66-1.18];0.3893

0.83[0.66-1.04];0.1098

0.83[0.50-1.39];0.4823

Platelet, $\times 10^{\wedge} 9 / \mathrm{L}$

Red blood count, $x 10^{\wedge} 12 / L$

0.93[0.86-1.01];0.0818

$1.001[1.000-$

$1.002] ; 0.2819$

1.002[1.001-

$1.003] ; 0.0015^{\star *}$

$1.002[0.999-$

$1.004] ; 0.1452$

Hematocrit, L/L

$0.30[0.11-0.80] ; 0.0163^{*}$

0.93[0.78-1.10];0.3688

0.91[0.79-1.06];0.2409

1.26[0.89-1.78];0.1925

0.75[0.09-6.67];0.7997

$0.18[0.03-1.13] ; 0.0671$

566.25[4.8865751.46];0.0090**

Renal and liver functions

* for $p \leq 0.05$, ** for $p \leq 0.01, * * *$ for $p \leq 0.001$; PD-1: Programmed death 1 inhibitors; PD-L1: programmed death 1 ligand inhibitors. 


\begin{tabular}{|c|c|c|c|c|}
\hline \multirow[t]{3}{*}{ Characteristics } & \multicolumn{2}{|l|}{ Before matching } & \multicolumn{2}{|l|}{ After 1:2 matching } \\
\hline & All-cause mortality & Composite outcome & All-cause mortality & Composite outcome \\
\hline & HR [95\% Cl];P value & HR [95\% Cl];P value & HR [95\% Cl];P value & HR [95\% Cl];P value \\
\hline K/Potassium, mmol/L & $0.91[0.80-1.03] ; 0.1211$ & $1.03[0.79-1.35] ; 0.8120$ & $0.87[0.69-1.09] ; 0.2157$ & $0.92[0.56-1.52] ; 0.7440$ \\
\hline Urate, $\mathrm{mmol} / \mathrm{L}$ & $1.54[0.72-3.30] ; 0.2671$ & $\begin{array}{l}5.04[0.95- \\
26.62] ; 0.0570\end{array}$ & $1.71[0.32-9.18] ; 0.5314$ & $0.65[0.02-19.52] ; 0.8040$ \\
\hline Albumin, $\mathrm{g} / \mathrm{L}$ & $\begin{array}{l}0.97[0.96-0.98] \\
<0.0001^{\star * \star}\end{array}$ & $0.98[0.96-1.00] ; 0.0540$ & $\begin{array}{l}0.98[0.96- \\
0.99] ; 0.0061^{\star \star}\end{array}$ & $1.04[0.99-1.09] ; 0.1011$ \\
\hline $\mathrm{Na} /$ Sodium, mmol/L & $\begin{array}{l}0.97[0.96-0.98] \\
<0.0001^{* \star *}\end{array}$ & $0.97[0.95-1.00] ; 0.0227^{\star}$ & $\begin{array}{l}0.95[0.92- \\
0.97] ; 0.0001^{\star \star \star}\end{array}$ & $0.97[0.91-1.05] ; 0.4786$ \\
\hline Urea, mmol/L & $0.99[0.97-1.02] ; 0.5540$ & $1.02[0.98-1.06] ; 0.4164$ & $0.98[0.93-1.03] ; 0.4672$ & $1.00[0.89-1.13] ; 0.9939$ \\
\hline Protein, g/L & $\begin{array}{l}0.99[0.99- \\
1.00] ; 0.0001 \star \star \star\end{array}$ & $1.00[0.99-1.01] ; 0.8901$ & $0.99[0.99-1.00] ; 0.0087^{* \star}$ & $1.00[0.98-1.02] ; 0.9227$ \\
\hline Bilirubin, umol/L & $\begin{array}{l}1.003[1.002-1.005] \\
<0.0001^{* * *}\end{array}$ & $1.00[1.00-1.01] ; 0.5730$ & $1.00[1.00-1.01] ; 0.9330$ & $1.00[0.99-1.01] ; 0.9730$ \\
\hline Creatinine, umol/L & $\begin{array}{l}1.001[1.000- \\
1.001] ; 0.2210\end{array}$ & $\begin{array}{l}1.000[0.998- \\
1.002] ; 0.7707\end{array}$ & $\begin{array}{l}1.001[0.999- \\
1.002] ; 0.2969\end{array}$ & $1.00[0.99-1.00] ; 0.4195$ \\
\hline SD of creatinine & $\begin{array}{l}1.001[1.000- \\
1.001] ; 0.0001^{\star \star \star}\end{array}$ & $\begin{array}{l}1.001[1.000- \\
1.002] ; 0.0719\end{array}$ & $\begin{array}{l}1.001[1.000- \\
1.001] ; 0.0974\end{array}$ & $\begin{array}{l}1.000[0.998- \\
1.002] ; 0.7760\end{array}$ \\
\hline Aspartate transaminase, $\mathrm{U} / \mathrm{L}$ & $\begin{array}{l}1.001[1.001-1.001] \\
<0.0001 * \star \star\end{array}$ & $\begin{array}{l}1.000[0.998- \\
1.002] ; 0.8846\end{array}$ & $\begin{array}{l}1.001[0.999- \\
1.002] ; 0.4383\end{array}$ & $0.99[0.98-1.01] ; 0.2798$ \\
\hline SD of aspartate transaminase & $\begin{array}{l}1.001[1.000-1.001] \\
<0.0001 * \star \star\end{array}$ & $\begin{array}{l}1.000[1.000- \\
1.001] ; 0.2117\end{array}$ & $\begin{array}{l}1.001[1.000- \\
1.002] ; 0.0155^{\star}\end{array}$ & $\begin{array}{l}1.001[0.997- \\
1.004] ; 0.6500\end{array}$ \\
\hline Alkaline phosphatase, U/L & $\begin{array}{l}1.001[1.001-1.002] \\
<0.0001^{* \star \star}\end{array}$ & $\begin{array}{l}1.000[0.999- \\
1.002] ; 0.7097\end{array}$ & $\begin{array}{l}1.000[0.999- \\
1.001] ; 0.4551\end{array}$ & $1.00[0.99-1.00] ; 0.2454$ \\
\hline SD of alkaline phosphatase & $\begin{array}{l}1.002[1.002-1.003] \\
<0.0001 * \star \star\end{array}$ & $\begin{array}{l}1.002[1.001- \\
1.003] ; 0.0014 * \star\end{array}$ & $\begin{array}{l}1.003[1.002-1.003] \\
<0.0001 * \star *\end{array}$ & $\begin{array}{l}1.002[1.000- \\
1.004] ; 0.0359 *\end{array}$ \\
\hline Alanine transaminase, $\mathrm{U} / \mathrm{L}$ & $\begin{array}{l}1.000[1.000- \\
1.001] ; 0.3737\end{array}$ & $\begin{array}{l}1.000[0.998- \\
1.002] ; 0.8684\end{array}$ & $\begin{array}{l}0.999[0.995- \\
1.002] ; 0.3897\end{array}$ & $0.99[0.98-1.00] ; 0.0914$ \\
\hline SD of alanine transaminase & $\begin{array}{l}1.001[1.001-1.002] \\
<0.0001^{\star \star \star}\end{array}$ & $\begin{array}{l}1.001[1.000- \\
1.002] ; 0.0177^{\star}\end{array}$ & $\begin{array}{l}1.001[1.000- \\
1.003] ; 0.0741\end{array}$ & $1.00[0.99-1.00] ; 0.5347$ \\
\hline \multicolumn{5}{|l|}{ Lipid, iron and calcium profile } \\
\hline Total iron-binding capacity, L & $1.00[0.98-1.02] ; 0.9971$ & $1.00[0.95-1.05] ; 0.9678$ & $0.99[0.95-1.04] ; 0.7518$ & $3.87[0.00-\operatorname{Inf}] ; 0.9993$ \\
\hline VitaminB12, pmol/L & $\begin{array}{l}0.999[0.998- \\
1.000] ; 0.1739\end{array}$ & $1.00[0.99-1.00] ; 0.1813$ & $\begin{array}{l}1.000[0.998- \\
1.002] ; 0.7853\end{array}$ & $1.00[0.99-1.01] ; 0.8806$ \\
\hline Folate, ng/mL & $1.00[0.97-1.03] ; 0.9877$ & $1.04[0.97-1.12] ; 0.2752$ & $1.00[0.96-1.05] ; 0.8835$ & $1.04[0.95-1.14] ; 0.3791$ \\
\hline Ferritin, pmol/L & $\begin{array}{l}1.000[1.000- \\
1.000] ; 0.4438\end{array}$ & $\begin{array}{l}1.000[1.000- \\
1.000] ; 0.3647\end{array}$ & $\begin{array}{l}1.000[1.000- \\
1.000] ; 0.2525\end{array}$ & $\begin{array}{l}1.000[1.000- \\
1.001] ; 0.1003\end{array}$ \\
\hline Calcium, mmol/L & $0.64[0.39-1.06] ; 0.0826$ & $0.41[0.13-1.26] ; 0.1212$ & $0.91[0.36-2.28] ; 0.8345$ & $1.40[0.20-9.84] ; 0.7350$ \\
\hline SD of calcium & $\begin{array}{l}12.70[5.21-30.97] \\
<0.0001^{* \star \star}\end{array}$ & $\begin{array}{l}2.10[0.13- \\
34.34] ; 0.6015\end{array}$ & $\begin{array}{l}4.69[1.00- \\
22.02] ; 0.0499 *\end{array}$ & $2.32[0.04-132.03] ; 0.6840$ \\
\hline Phosphate, mmol/L & $0.83[0.59-1.18] ; 0.3059$ & $0.79[0.36-1.74] ; 0.5548$ & $0.98[0.50-1.90] ; 0.9464$ & $0.61[0.14-2.69] ; 0.5115$ \\
\hline SD of phosphate & $\begin{array}{l}5.97[3.23-11.04] \\
<0.0001^{\star \star \star}\end{array}$ & $\begin{array}{l}\text { 7.44[1.81- } \\
30.57] ; 0.0054 \star \star\end{array}$ & $\begin{array}{l}3.91[0.74- \\
20.76] ; 0.1098\end{array}$ & $\begin{array}{l}\text { 18.22[0.41- } \\
815.46] ; 0.1345\end{array}$ \\
\hline Inorganic, mmol/L & $1.01[1.00-1.03] ; 0.0836$ & $0.99[0.94-1.04] ; 0.7433$ & $\begin{array}{l}1.04[1.01- \\
1.08] ; 0.0055^{\star \star}\end{array}$ & $0.97[0.84-1.13] ; 0.7058$ \\
\hline
\end{tabular}

* for $p \leq 0.05$, ** for $p \leq 0.01, * \star *$ for $p \leq 0.001$; PD-1: Programmed death 1 inhibitors; PD-L1: programmed death 1 ligand inhibitors. 


\begin{tabular}{|c|c|c|c|c|}
\hline \multirow[t]{3}{*}{ Characteristics } & \multicolumn{2}{|l|}{ Before matching } & \multicolumn{2}{|l|}{ After 1:2 matching } \\
\hline & All-cause mortality & Composite outcome & All-cause mortality & Composite outcome \\
\hline & HR [95\% Cl];P value & HR [95\% Cl];P value & HR [95\% Cl];P value & HR [95\% Cl];P value \\
\hline SD of inorganic & $1.00[0.95-1.07] ; 0.8984$ & $0.98[0.82-1.17] ; 0.8125$ & $1.08[0.85-1.36] ; 0.5374$ & $0.09[0.00-21.93] ; 0.3871$ \\
\hline \multicolumn{5}{|l|}{ Glycemic and clotting profile } \\
\hline Triglyceride, $\mathrm{mmol} / \mathrm{L}$ & $0.96[0.88-1.05] ; 0.3264$ & $0.74[0.56-0.99] ; 0.0436^{*}$ & $0.90[0.78-1.04] ; 0.1556$ & $0.61[0.35-1.09] ; 0.0933$ \\
\hline SD of triglyceride & $0.84[0.64-1.11] ; 0.2196$ & $0.20[0.04-1.02] ; 0.0526$ & $0.69[0.41-1.17] ; 0.1655$ & $0.54[0.08-3.53] ; 0.5213$ \\
\hline Glucose, $\mathrm{mmol} / \mathrm{L}$ & $1.02[0.99-1.04] ; 0.1738$ & $1.00[0.94-1.05] ; 0.9179$ & $0.99[0.95-1.03] ; 0.5861$ & $0.90[0.79-1.04] ; 0.1558$ \\
\hline SD of glucose & $\begin{array}{l}1.06[1.02- \\
1.11] ; 0.0032^{\star *}\end{array}$ & $1.07[0.98-1.16] ; 0.1337$ & $1.07[1.00-1.14] ; 0.0580$ & 1.07[0.92-1.23];0.3816 \\
\hline $\mathrm{HbA} 1 \mathrm{c}, \mathrm{g} / \mathrm{dL}$ & $0.97[0.95-1.00] ; 0.0539$ & $1.00[0.94-1.06] ; 0.9969$ & $0.95[0.91-1.00] ; 0.0543$ & $\begin{array}{l}1.19[1.05- \\
1.34] ; 0.0072^{\star \star}\end{array}$ \\
\hline $\mathrm{SD}$ of $\mathrm{HbA} 1 \mathrm{c}$ & $\begin{array}{l}1.58[1.44-1.74] \\
<0.0001^{\star * \star}\end{array}$ & $\begin{array}{l}1.51[1.23- \\
1.85] ; 0.0001^{\star \star \star}\end{array}$ & $\begin{array}{l}1.52[1.29-1.79] \\
<0.0001^{\star \star \star}\end{array}$ & $\begin{array}{l}1.94[1.36- \\
2.76] ; 0.0003^{\star \star \star}\end{array}$ \\
\hline High sensitive troponin-l, ng/L & $\begin{array}{l}1.000[1.000- \\
1.000] ; 0.9239\end{array}$ & $\begin{array}{l}1.000[1.000- \\
1.000] ; 0.1181\end{array}$ & $\begin{array}{l}1.000[1.000- \\
1.000] ; 0.9382\end{array}$ & $\begin{array}{l}1.000[1.000- \\
1.001] ; 0.1785\end{array}$ \\
\hline SD of high sensitive troponin-I & $\begin{array}{l}1.000[1.000- \\
1.000] ; 0.7182\end{array}$ & $\begin{array}{l}1.000[1.000- \\
1.000] ; 0.0044^{\star *}\end{array}$ & $\begin{array}{l}1.000[0.999- \\
1.001] ; 0.9978\end{array}$ & $\begin{array}{l}1.000[1.000- \\
1.001] ; 0.2563\end{array}$ \\
\hline APTT, second & $1.01[1.00-1.03] ; 0.1426$ & $0.98[0.93-1.03] ; 0.3733$ & $0.99[0.95-1.03] ; 0.6325$ & $0.98[0.89-1.08] ; 0.6750$ \\
\hline SD of APTT & $\begin{array}{l}1.05[1.02- \\
1.08] ; 0.0006 \star \star \star\end{array}$ & $0.99[0.90-1.10] ; 0.8880$ & $1.01[0.90-1.13] ; 0.8927$ & $1.10[0.86-1.42] ; 0.4432$ \\
\hline Lactate dehydrogenase, $\mathrm{U} / \mathrm{L}$ & $\begin{array}{l}1.000[1.000-1.000] \\
<0.0001 * * *\end{array}$ & $\begin{array}{l}1.000[1.000- \\
1.001] ; 0.0165^{*}\end{array}$ & $\begin{array}{l}1.000[1.000- \\
1.001] ; 0.0001^{\star \star \star}\end{array}$ & $\begin{array}{l}1.000[0.999- \\
1.001] ; 0.6208\end{array}$ \\
\hline SD of lactate dehydrogenase & $\begin{array}{l}1.000[1.000-1.000] \\
<0.0001^{* * *}\end{array}$ & $\begin{array}{l}1.000[1.000- \\
1.001] ; 0.0229^{*}\end{array}$ & $\begin{array}{l}1.002[1.001-1.002] \\
<0.0001^{\star \star \star}\end{array}$ & $\begin{array}{l}1.002[1.000- \\
1.003] ; 0.0056^{\star *}\end{array}$ \\
\hline Total cholesterol, $\mathrm{mmol} / \mathrm{L}$ & $0.98[0.90-1.07] ; 0.6769$ & $0.92[0.75-1.13] ; 0.4335$ & $1.05[0.90-1.22] ; 0.5598$ & $1.42[1.00-2.00] ; 0.0490^{*}$ \\
\hline SD of total cholesterol & $1.14[0.84-1.55] ; 0.3905$ & $0.93[0.42-2.07] ; 0.8677$ & $1.23[0.73-2.07] ; 0.4394$ & $2.73[0.81-9.19] ; 0.1058$ \\
\hline Low-density lipoprotein, mmol/L & $1.00[0.90-1.11] ; 0.9905$ & $0.96[0.76-1.22] ; 0.7514$ & $1.14[0.96-1.37] ; 0.1398$ & $\begin{array}{l}1.67[1.14- \\
2.45] ; 0.0091^{* *}\end{array}$ \\
\hline SD of low-density lipoprotein & $1.22[0.84-1.78] ; 0.2952$ & $1.13[0.45-2.83] ; 0.7919$ & $1.66[0.77-3.55] ; 0.1928$ & $\begin{array}{l}\text { 7.64[1.84- } \\
31.77] ; 0.0051^{\star *}\end{array}$ \\
\hline High-density lipoprotein, $\mathrm{mmol} / \mathrm{L}$ & $0.93[0.73-1.19] ; 0.5774$ & $1.27[0.77-2.11] ; 0.3530$ & $0.97[0.62-1.50] ; 0.8772$ & $1.96[0.82-4.71] ; 0.1309$ \\
\hline SD of high-density lipoprotein & $2.42[0.85-6.87] ; 0.0971$ & $\begin{array}{l}1.55[0.11- \\
21.84] ; 0.7461\end{array}$ & $1.32[0.19-9.12] ; 0.7758$ & $1.09[0.01-164.28] ; 0.9719$ \\
\hline
\end{tabular}

\section{Sensitivity analysis}

Supplementary Table 7 presented the adjusted hazard ratios (and 95\% Cls) of PD-L1 v.s. PD-1 with cause-specific and subdistribution hazard competing risk analysis models for new onset cardiac composite and mortality outcomes after 1:2 propensity score matching. Supplementary Table 8 presented the hazard ratios for associations of PD-L1 v.s. PD-1 using Cox proportional hazard model for adverse new onset cardiac composite and mortality outcome in the 1:2 matched cohort, with half-year lag time. Supplementary Table 9 presented the risk of incident new onset cardiac composite and mortality outcomes associated with treatment of PD-L1 v.s. PD-1 with multiple matching adjustment approaches including propensity score stratification, high-dimensional propensity score matching, and propensity score matching with inverse probability of treatment weighting. The above analysis confirmed the protective effects of PD-L1 treatment over PD-1 treatment on new onset cardiac complications and mortality risks $(H R<1, P$ value $<0.05)$.

\section{Prediction strength of subclinical inflammatory biomarkers}

In the matched cohort, higher aspartate transaminase-to-alanine transaminase ratio (HR: 1.09, 95\% Cl: [1.04-1.14], P value = 0.0004) and higher monocyte-tolymphocyte ratio (HR: 1.2, 95\% Cl: [1.02-1.43], P value $=0.0305)$ were significantly associated with all-cause mortality. A higher urea-to-creatinine ratio (HR: 1.01, 95\% Cl: [1.00-1.01], P value $=0.0007)$ was significantly associated with new onset cardiac composite outcome (Table 4). The boxplots of inflammatory biomarkers stratified by PD-1/PD-L1 inhibitor use and development of adverse outcomes are shown in Fig. 4.

\section{Discussion}


The main findings are that: i) the incidence of cardiovascular complications after PD-1 or PD-L1 inhibitor use was $16 \%$ in this territory-wide cohort of Chinese patients from Hong Kong, ii) multivariate Cox regression showed older age, a shorter average readmission interval and a higher number of hospital admissions were significant predictors of cardiovascular complications and iii) patients who developed cardiovascular complications had shorter average readmission interval and higher number of hospitalizations after treatment with PD-1/PD-L1 inhibitors.

Cardiac involvement in PD1 or PD-L1 inhibitors is variable, and can potentially affect the conduction system, myocardium or pericardium (16). Thus, heart block (17), Takotsubo cardiomyopathy $(18)$, myocarditis $(19,20)$ and pericarditis $(21)$ have been reported. A meta-analysis performed in 2018 found that antiPD-1/PD-L1-related fatalities were often from pneumonitis (333 [35\%]), hepatitis (115 [22\%]), and neurotoxic effects (50 [15\%]). Combination PD-1/ cytotoxic T-lymphocyte-associated protein 4 (CTLA-4) deaths were frequently from colitis (32 [37\%]) and myocarditis (22 [25\%]) (22). In an analysis of the World Health Organization global database of adverse drug reactions in 2019, $2.1 \%$ of 106025 patients receiving PD-1 or PD-L1 inhibitors had cardiovascular complications (8). However, previous studies have largely been limited to case reports $(17,23)$, case series $(24)$, single-center studies $(25)$ or small registries $(26,27)$. In this territory-wide study from Hong Kong, we found that cardiovascular complications occurred in 16\% of all patients receiving PD1 or PD-L1 inhibitors. Of these, the commonest is heart failure. Previously, acute heart failure has been described in the context of myocarditis (28), but heart failure without myocarditis has also been reported (29). Our study also identified cases of acute myocardial infarction following the initiation of PD-1/PD-L1 inhibitor therapy. Such findings would be in keeping with coronary toxicity that has been reported in the context of PD-1 inhibitor therapy (26).

Interestingly, our study did not identify any patients with myocarditis after treatment with PD-1 or PD-L1 inhibitors. Moreover, within the excluded patients with prior cardiovascular complications, none developed subsequent myocarditis. In 964 patients attending Massachusetts General Hospital, the incidence of myocarditis was $1.1 \%(n=35)(27)$. In this cohort, myocarditis was more frequently observed in patients with pre-existing cardiovascular comorbidities. Nevertheless, another study using the VigiBase database found 101 cases of severe myocarditis, of which $75 \%$ of the myocarditis cases did not have preexisting cardiovascular disease (30). A single-center study of 283 patients from China found only 3 cases (1.1\%) of myocarditis, with variable presentations such as palpitations, dyspnea, and fatigue, or asymptomatic with incidental finding of grade 3 atrioventricular block and premature ventricular complexes on the electrocardiogram (25). In a pooled, retrospective review of three trials including 448 patients with advanced melanoma receiving PD-1/PD-L1 inhibitor therapy, no cases of myocarditis were identified (31). In association with myocarditis, different investigators have reported the presence of conduction abnormalities in the form of atrioventricular block $(17,24,25,32)$.

\section{Limitations}

There are some limitations of this study that should be acknowledged. Firstly, this was an administrative database study, and therefore cancer staging details could not be extracted. Secondly, under-coding or miscoding remains a possibility as with studies of a similar nature.

\section{Conclusions}

Compared with PD-1 inhibitor use, PD-L1 inhibitor use was significantly associated with lower risk of new onset composite outcome and all-cause mortality both before and after 1:1 propensity score matching. Patients who developed cardiovascular complications had shorter average readmission intervals and a higher number of hospitalizations after treatment with PD-1/PD-L1 inhibitors both before and after 1:1 propensity score matching.

\section{Declarations}

\section{Consent for publication}

N/A.

\section{Availability of data and material}

Data availability upon request to the corresponding author.

\section{Competing interests}

None.

\section{Funding}

None.

\section{Authors' contributions}

JZ, SL, GT: data analysis, data interpretation, statistical analysis, manuscript drafting, critical revision of manuscript

IL, SL, LY, TL, YX, YZ: data interpretation, manuscript drafting

EWC, ICKW: project planning, data acquisition, data interpretation, critical revision of manuscript

QZ: study conception, study supervision, project planning, data interpretation, statistical analysis, manuscript drafting, critical revision of manuscript 
None.

\section{References}

1. Kalinich M, Murphy W, Wongvibulsin S, et al. Prediction of severe immune-related adverse events requiring hospital admission in patients on immune checkpoint inhibitors: study of a population level insurance claims database from the USA. J Immunother Cancer 2021;9.

2. Griewing LM, Schweizer C, Schubert P, et al. Questionnaire-based detection of immune-related adverse events in cancer patients treated with PD-1/PD-L1 immune checkpoint inhibitors. BMC Cancer. 2021;21:314.

3. Wu C, Zhong L, Wu Q, Lin S, Xie X. The safety and efficacy of immune-checkpoint inhibitors in patients with cancer and pre-existing autoimmune diseases. Immunotherapy. 2021;13:527-39.

4. Kang JH, Bluestone JA, Young A. Predicting and Preventing Immune Checkpoint Inhibitor Toxicity: Targeting Cytokines. Trends Immunol. 2021;42:293311.

5. Lee S, Tse G. A Patient with Atezolizumab-Induced Autoimmune Diabetes Mellitus Presenting with Diabetic Ketoacidosis. Cardiovascular Innovations and Applications; 2021

6. Zhang N, Tse G, Liu T. Neutrophil-lymphocyte ratio in the immune checkpoint inhibitors-related atherosclerosis. Eur Heart J 2021.

7. Ball S, Ghosh RK, Wongsaengsak S, et al. Cardiovascular Toxicities of Immune Checkpoint Inhibitors: JACC Review Topic of the Week. J Am Coll Cardiol. 2019;74:1714-27.

8. Upadhrasta S, Elias H, Patel K, Zheng L. Managing cardiotoxicity associated with immune checkpoint inhibitors. Chronic Dis Transl Med. $2019 ; 5: 6-14$.

9. Muller OJ, SpehImann ME, Frey N. Cardio-toxicity of checkpoint inhibitors. J Thorac Dis. 2018;10:4400-4.

10. Lee S, Zhou J, Guo CL, et al. Predictive scores for identifying patients with type 2 diabetes mellitus at risk of acute myocardial infarction and sudden cardiac death. Endocrinol Diabetes Metab. 2021;4:e00240.

11. Tse G, Zhou J, Lee S, et al. Relationship between angiotensin-converting enzyme inhibitors or angiotensin receptor blockers and COVID-19 incidence or severe disease. J Hypertens. 2021;39:1717-24.

12. Lee S, Zhou J, Leung KSK, et al. Development of a predictive risk model for all-cause mortality in patients with diabetes in Hong Kong. BMJ Open Diabetes Res Care 2021;9.

13. Austin PC. An Introduction to Propensity Score Methods for Reducing the Effects of Confounding in Observational Studies. Multivariate Behav Res. 2011;46:399-424.

14. Austin PC, Stuart EA. Moving towards best practice when using inverse probability of treatment weighting (IPTW) using the propensity score to estimate causal treatment effects in observational studies. Stat Med. 2015;34:3661-79.

15. Schneeweiss S, Rassen JA, Glynn RJ, Avorn J, Mogun H, Brookhart MA. High-dimensional propensity score adjustment in studies of treatment effects using health care claims data. Epidemiology. 2009;20:512-22.

16. Lyon AR, Yousaf N, Battisti NML, Moslehi J, Larkin J. Immune checkpoint inhibitors and cardiovascular toxicity. Lancet Oncol. 2018;19:e447-58.

17. Khan A, Riaz S, Carhart R. Jr. Pembrolizumab-Induced Mobitz Type 2 Second-Degree Atrioventricular Block. Case Rep Cardiol. 2020;2020:8428210.

18. Geisler BP, Raad RA, Esaian D, Sharon E, Schwartz DR. Apical ballooning and cardiomyopathy in a melanoma patient treated with ipilimumab: a case of takotsubo-like syndrome. J Immunother Cancer. 2015;3:4.

19. Norwood TG, Westbrook BC, Johnson DB, et al. Smoldering myocarditis following immune checkpoint blockade. J Immunother Cancer. $2017 ; 5: 91$.

20. Semper H, Muehlberg F, Schulz-Menger J, Allewelt M, Grohe C. Drug-induced myocarditis after nivolumab treatment in a patient with PDL1- negative squamous cell carcinoma of the lung. Lung Cancer. 2016;99:117-9.

21. de Almeida DVP, Gomes JR, Haddad FJ, Buzaid AC. Immune-mediated Pericarditis With Pericardial Tamponade During Nivolumab Therapy. J Immunother. 2018;41:329-31.

22. Wang DY, Salem JE, Cohen JV, et al. Fatal Toxic Effects Associated With Immune Checkpoint Inhibitors: A Systematic Review and Meta-analysis. JAMA Oncol. 2018;4:1721-8.

23. Johnson DB, Balko JM, Compton ML, et al. Fulminant Myocarditis with Combination Immune Checkpoint Blockade. N Engl J Med. 2016;375:1749-55.

24. Heinzerling L, Ott PA, Hodi FS, et al. Cardiotoxicity associated with CTLA4 and PD1 blocking immunotherapy. J Immunother Cancer. 2016;4:50.

25. Wang F, Sun X, Qin S, et al. A retrospective study of immune checkpoint inhibitor-associated myocarditis in a single center in China. Chin Clin Oncol. 2020;9:16.

26. Ferreira M, Pichon E, Carmier D, et al. Coronary Toxicities of Anti-PD-1 and Anti-PD-L1 Immunotherapies: a Case Report and Review of the Literature and International Registries. Target Oncol. 2018;13:509-15.

27. Mahmood SS, Fradley MG, Cohen JV, et al. Myocarditis in Patients Treated With Immune Checkpoint Inhibitors. J Am Coll Cardiol. 2018;71:1755-64.

28. Laubli H, Balmelli C, Bossard M, Pfister O, Glatz K, Zippelius A. Acute heart failure due to autoimmune myocarditis under pembrolizumab treatment for metastatic melanoma. J Immunother Cancer. 2015;3:11.

29. Chauhan A, Burkeen G, Houranieh J, Arnold S, Anthony L. Immune checkpoint-associated cardiotoxicity: case report with systematic review of literature. Ann Oncol. 2017;28:2034-8.

30. Moslehi JJ, Salem JE, Sosman JA, Lebrun-Vignes B, Johnson DB. Increased reporting of fatal immune checkpoint inhibitor-associated myocarditis. Lancet. 2018;391:933. 
31. Sznol M, Ferrucci PF, Hogg D, et al. Pooled Analysis Safety Profile of Nivolumab and Ipilimumab Combination Therapy in Patients With Advanced Melanoma. J Clin Oncol. 2017;35:3815-22.

32. Lopez EM, Dunn S, Mazimba S. MALIGNANT ARRHYTHMIAS IN, AUTOIMMUNE MYOCARDITIS SECONDARY TO IMMUNE CHECKPOINT BLOCKADE TREATMENT. J Am Coll Cardiol. 2018;71:A2375.

\section{Figures}

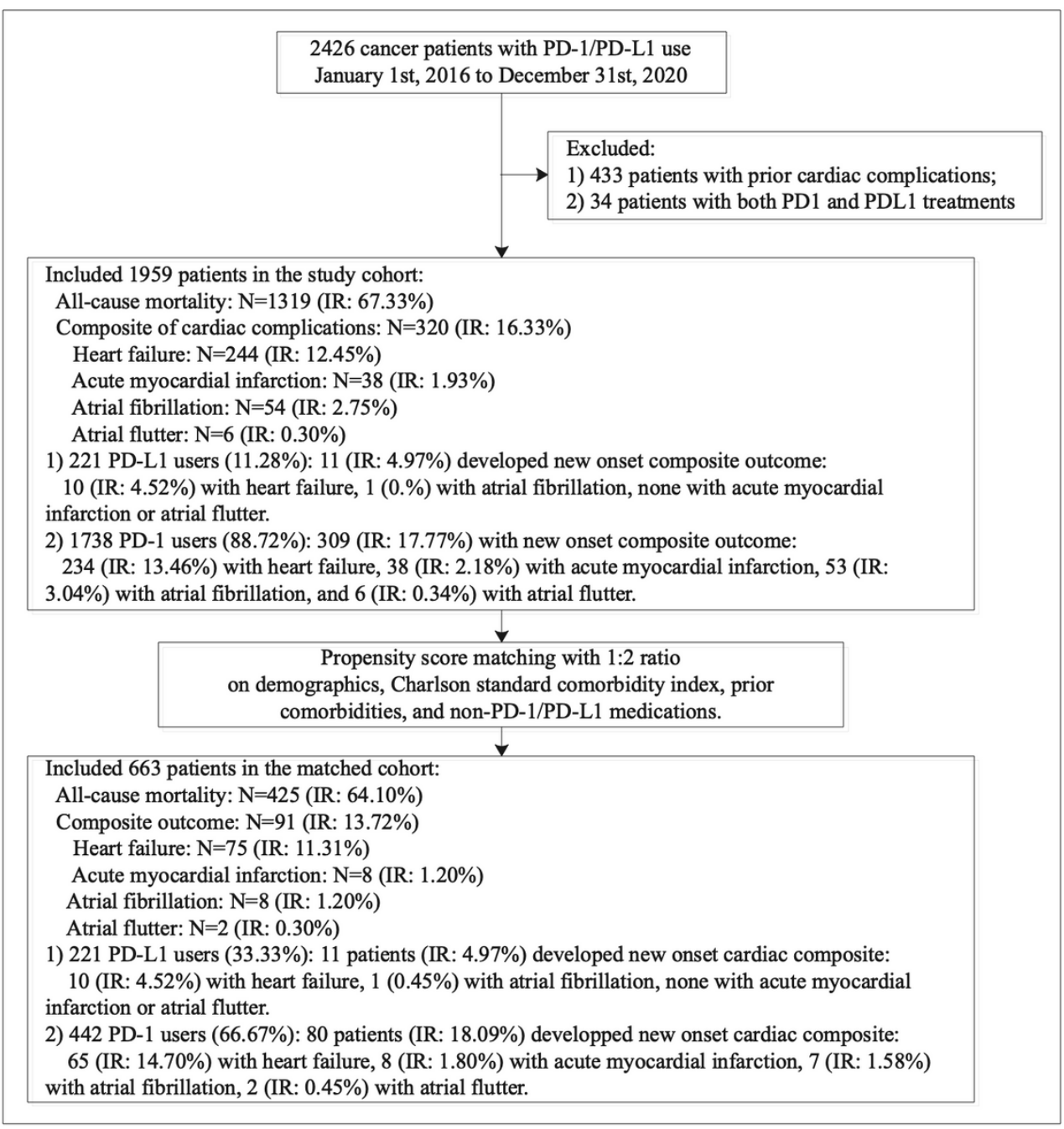

\section{Figure 1}

IR: Incidence rate; PD-1: Programmed death 1 inhibitors; PD-L1: programmed death 1 ligand inhibitors. Study flow diagram describing derivation of the study cohort. 

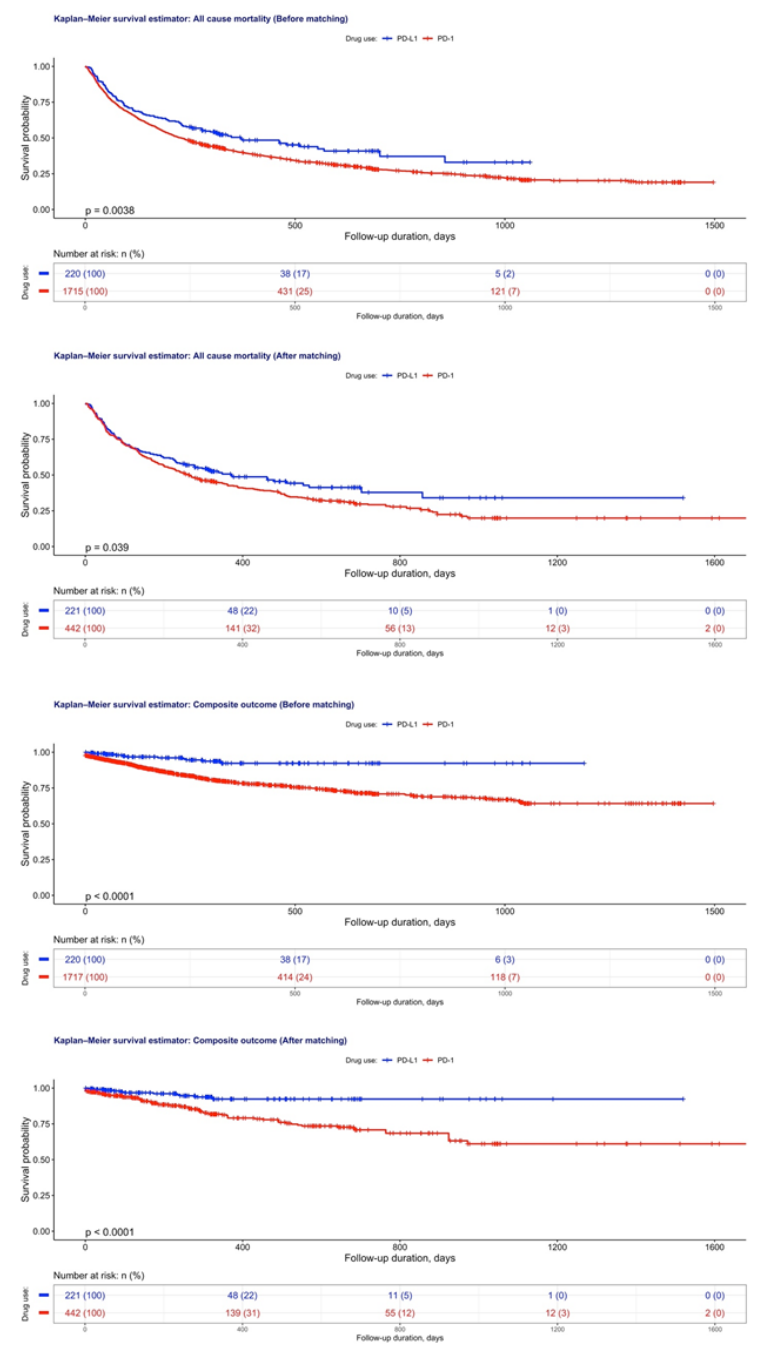

\section{Figure 2}

Kaplan-Meier survival curves of new onset cardiac complications and all-cause mortality in cancer patients stratified by PD-1 or PD-L1 inhibitor use before and after 1:2 propensity score matching. 

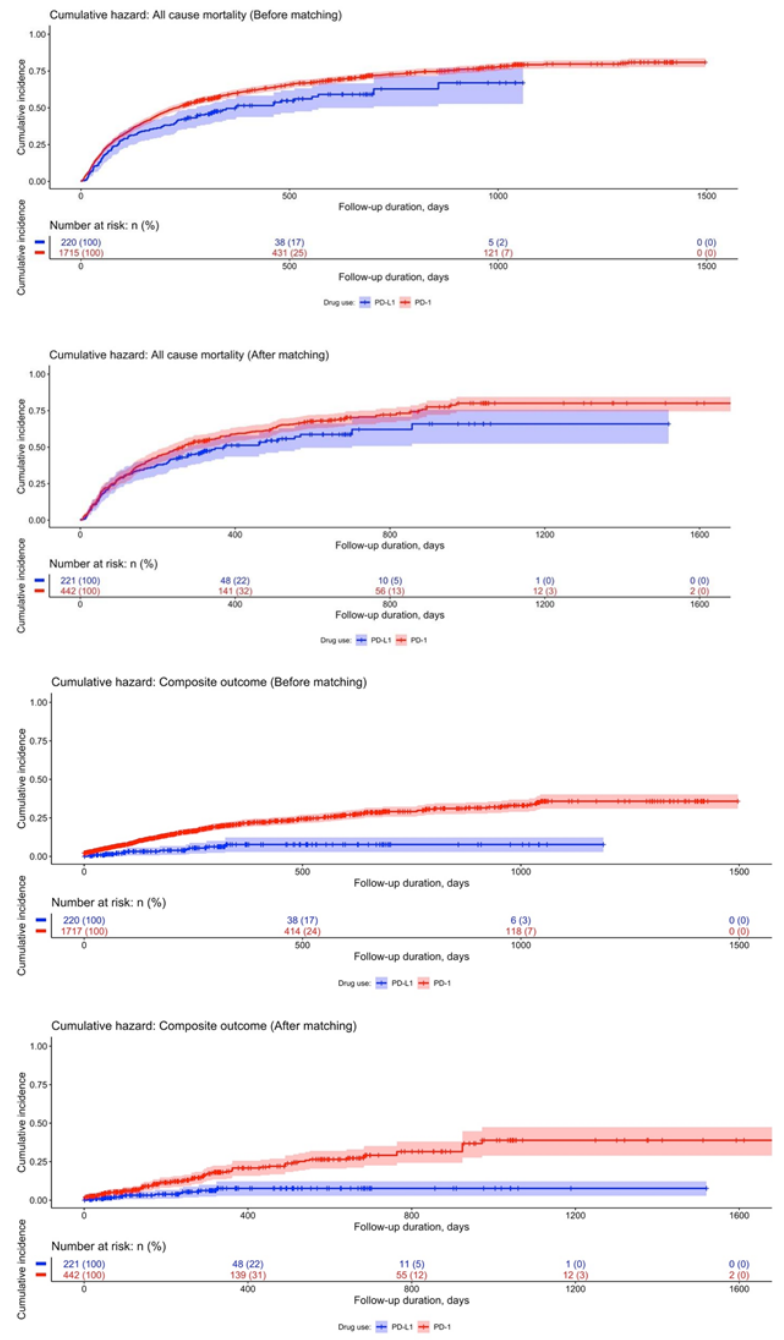

\section{Figure 3}

Cumulative incidence curves of new onset cardiac complications and all-cause mortality in cancer patients stratified by PD-1 or PD-L1 inhibitor use before and after 1:2 propensity score matching. 

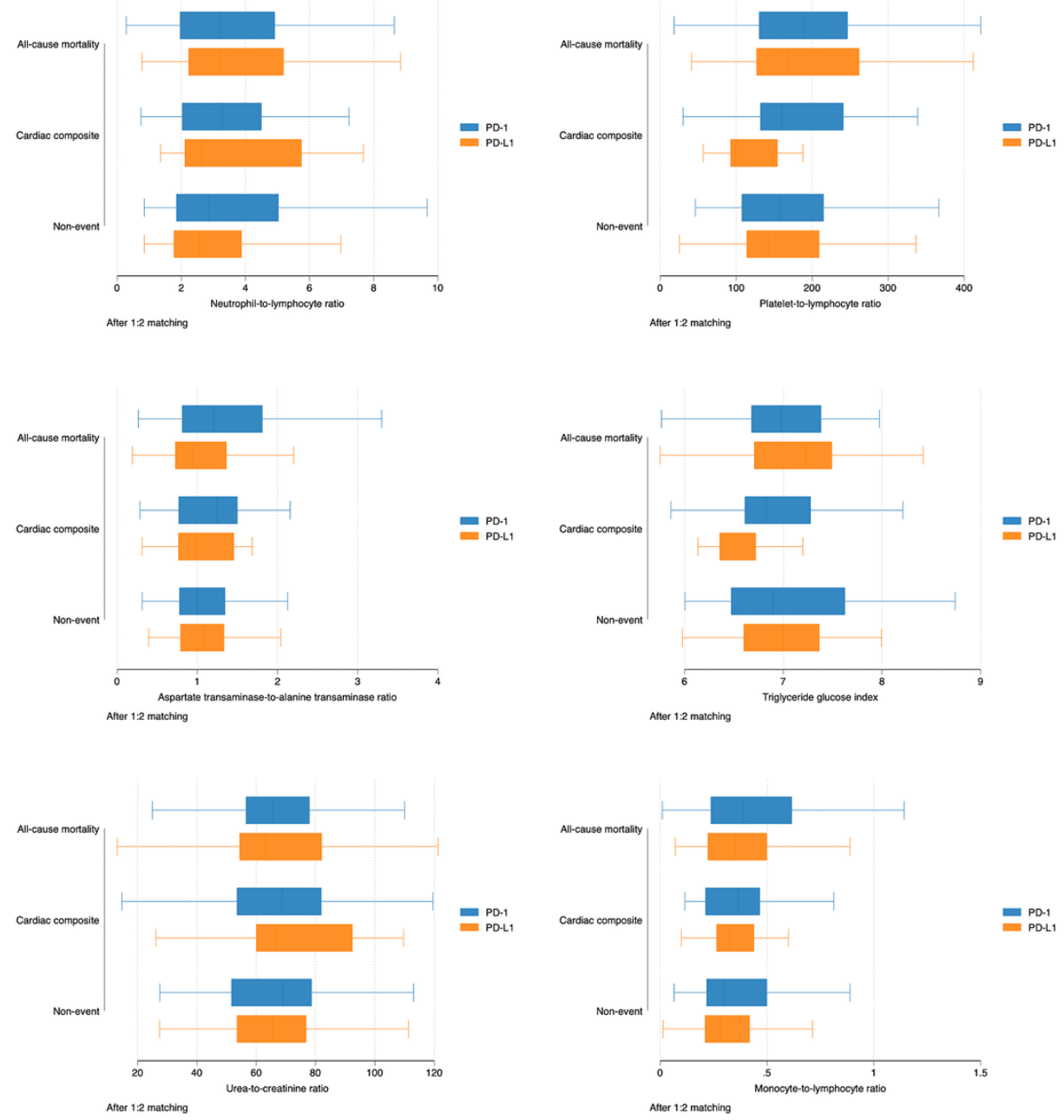

\section{Figure 4}

Boxplots of subclinical inflammatory biomarkers stratified by PD-1/PD-L1 inhibitor use and adverse events development in the matched cohort. 

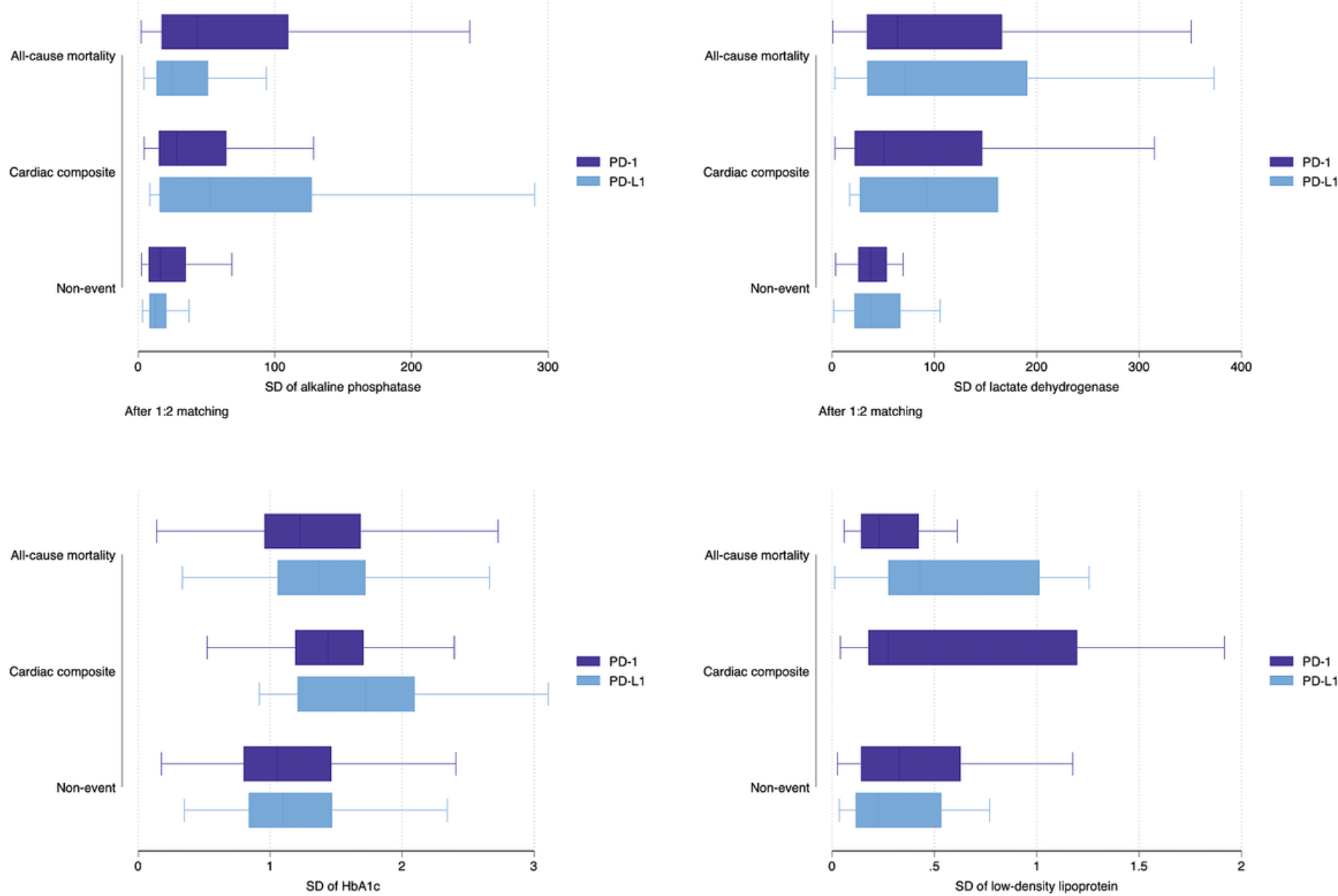

After 1:2 matching

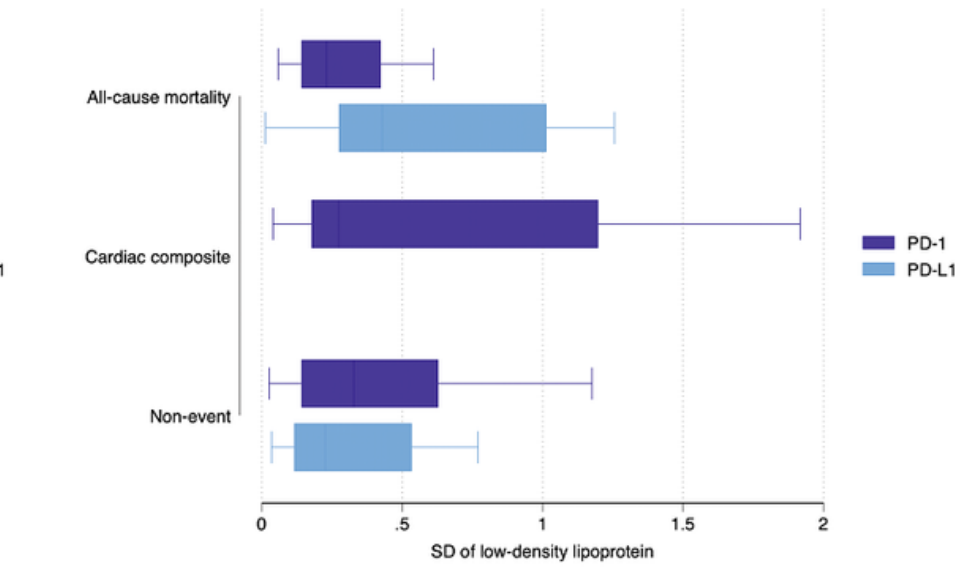

After 1:2 matching

\section{Figure 5}

Boxplots of significant laboratory examination variability to predict adverse outcomes stratified by PD-1/PD-L1 inhibitor treatment in the matched cohort.

\section{Supplementary Files}

This is a list of supplementary files associated with this preprint. Click to download.

- Supplementarymaterialscardiac20210813.docx 